\title{
Galois representations on the cohomology of hyper-Kähler varieties
}

\author{
Salvatore Floccari ${ }^{1}$
}

Received: 11 May 2021 / Accepted: 7 November 2021 / Published online: 4 January 2022

(c) The Author(s) 2022

\begin{abstract}
We show that the André motive of a hyper-Kähler variety $X$ over a field $K \subset \mathbb{C}$ with $b_{2}(X)>6$ is governed by its component in degree 2 . More precisely, we prove that if $X_{1}$ and $X_{2}$ are deformation equivalent hyper-Kähler varieties with $b_{2}\left(X_{i}\right)>6$ and if there exists a Hodge isometry $f: H^{2}\left(X_{1}, \mathbb{Q}\right) \rightarrow H^{2}\left(X_{2}, \mathbb{Q}\right)$, then the André motives of $X_{1}$ and $X_{2}$ are isomorphic after a finite extension of $K$, up to an additional technical assumption in presence of non-trivial odd cohomology. As a consequence, the Galois representations on the étale cohomology of $X_{1}$ and $X_{2}$ are isomorphic as well. We prove a similar result for varieties over a finite field which can be lifted to hyper-Kähler varieties for which the Mumford-Tate conjecture is true.
\end{abstract}

Keywords Hyper-Kähler varieties · Motives · Hodge theory · Galois representations

Mathematics Subject Classification $14 \mathrm{C} 30 \cdot 14 \mathrm{~F} 20 \cdot 14 \mathrm{~J} 20 \cdot 14 \mathrm{~J} 32$

\section{Introduction}

A guiding principle in the study of hyper-Kähler manifolds is that many of their geometric properties are governed by their cohomology in degree 2. Perhaps the most spectacular illustration of this principle is the global Torelli theorem due to Huybrechts, Markman and Verbitsky, which precisely explains to what extent the birational class of a hyper-Kähler manifold $X$ can be recovered from the integral Hodge structure on the lattice $H^{2}(X, \mathbb{Z})$.

As another example, it is known that the total Hodge structure on the rational singular cohomology $H_{X}^{*}:=H^{*}(X, \mathbb{Q})$ is determined by the Hodge structure on $H_{X}^{2}$. This fact is a consequence of the properties of the Looijenga-Lunts-Verbitsky Lie algebra that was introduced in [18,30]; a complete proof of this result has been given by Soldatenkov in [27].

Let now $K \subset \mathbb{C}$ be a subfield which is finitely generated over $\mathbb{Q}$ and let $X$ be a hyperKähler variety over $K$. We fix a prime number $\ell$ and consider the étale cohomology groups $H_{X, \ell}^{*}:=H_{\mathrm{et}}^{*}\left(X_{\bar{K}}, \mathbb{Q}_{\ell}\right)$ of $X$. It is then natural to ask whether the Galois representation on the

Salvatore Floccari

floccari@math.uni-hannover.de

1 Institute of Algebraic Geometry, Leibniz University Hannover, Hannover, Germany 
$\ell$-adic cohomology $H_{X, \ell}^{*}$ of $X$ is determined by its restriction to $H_{X, \ell}^{2}$. Going even further, we may ask the analogous question at the level of homological or Chow motives-however, in this setting the existence of Künneth components is not known in general.

To circumvent this difficulty, we will work with André's category of motives [2].

We prove that, up to a finite field extension of the base field, the total André motive $\mathcal{H}_{X}^{*}=\bigoplus_{j} \mathcal{H}_{X}^{j}$ of a hyper-Kähler variety $X$ is governed by its component in degree 2 . In what follows we say that two hyper-Kähler varieties $X_{1}$ and $X_{2}$ over a field $K \subset \mathbb{C}$ are deformation equivalent if the complex varieties $X_{1, \mathbb{C}}$ and $X_{2, \mathbb{C}}$ are deformation equivalent in the analytic sense. We let $H_{X_{i}}^{*}:=\bigoplus_{j} H^{j}\left(X_{i, \mathbb{C}}, \mathbb{Q}\right)$.

Theorem 1.1 (= Theorem 4.7) Let $X_{1}, X_{2}$ be deformation equivalent hyper-Kähler varieties with $b_{2}\left(X_{i}\right)>6$ over a field $K \subset \mathbb{C}$. If $X_{i}$ has non-trivial cohomology in odd degree, assume that, for $i=1,2$, the motive $\mathcal{H}_{A_{i}}^{1}$ belongs to the tannakian category generated by $\mathcal{H}_{X_{i}}^{*}$, where $A_{i}$ is the Kuga-Satake variety on $H_{X_{i}}^{2}$. Assume that there exists a Hodge isometry $f: H_{X_{1}}^{2} \rightarrow H_{X_{2}}^{2}$. Then, there exist a finite field extension $K^{\prime} / K$ and an isomorphism of graded algebras $F: H_{X_{1}}^{*} \rightarrow H_{X_{2}}^{*}$ which is the realization of an isomorphism of André motives $\mathcal{H}_{X_{1, K^{\prime}}}^{*} \rightarrow \mathcal{H}_{X_{2, K^{\prime}}}^{*}$ over $K^{\prime}$.

The map $F$ obtained in the theorem is in particular an isomorphism of Hodge structures. The assumption on the second Betti number is needed to show that deformation equivalent hyper-Kähler varieties can be connected via a chain of polarized deformations; see Theorem 5.1 for the precise statement. All known hyper-Kähler varieties have $b_{2} \geq 7$.

In presence of non-trivial odd cohomology we need an extra assumption to control the odd part of the motive. The Kuga-Satake variety $A_{i}$ is an abelian variety closely related to $H_{X_{i}}^{2}$ [8]; we expect that its motive belongs to the tannakian category generated by $H_{X_{i}}^{*}$. By [10] this happens for the known hyper-Kähler varieties with non-trivial odd cohomology, which are those of generalized Kummer deformation type. We obtain the following consequence of Theorem 1.1.

Corollary 1.2 (=Corollary 4.8) Let $K \subset \mathbb{C}$ be a subfield which is finitely generated over $\mathbb{Q}$ and let $X_{1}, X_{2}$ be deformation equivalent hyper-Kähler varieties over $K$ such that $b_{2}\left(X_{i}\right)>6$. If $X_{i}$ has non-trivial cohomology in odd degree, assume further that, for $i=1,2$, the motive $\mathcal{H}_{A_{i}}^{1}$ belongs to the tannakian category generated by $\mathcal{H}_{X_{i}}^{*}$, where $A_{i}$ is the Kuga-Satake variety on $H_{X_{i}}^{2}$. Assume that there exists a $\operatorname{Gal}(\bar{K} / K)$-equivariant isometry $f: H_{X_{1}, \ell}^{2} \rightarrow H_{X_{2}, \ell}^{2}$. Then, there exist a finite field extension $K^{\prime} / K$ and a $\operatorname{Gal}\left(\bar{K} / K^{\prime}\right)$-equivariant isomorphism of graded algebras $F: H_{X_{1}, \ell}^{*} \rightarrow H_{X_{2}, \ell}^{*}$.

The corollary is the $\ell$-adic counterpart of the Hodge theoretic result from [27]. This is not surprising: if the Mumford-Tate conjecture was true, the corollary would be a direct consequence of its analogue in Hodge theory. Even though the Mumford-Tate conjecture is not known for arbitrary hyper-Kähler varieties, in [10] we proved it for all hyper-Kähler varieties of known deformation type. When the conjecture holds, we obtain a more precise result on the Galois representations $H_{X_{i, \ell}}^{*}$. Let us say that two hyper-Kähler varieties $X_{1}$ and $X_{2}$ are $H_{\ell}^{*}$-equivalent if there exists an isomorphism of graded algebras $H_{X_{1}, \ell}^{*} \cong H_{X_{2}, \ell}^{*}$ which restricts to an isometry in degree 2 with respect to the Beauville-Bogomolov pairings. Deformation equivalent $X_{1}$ and $X_{2}$ are $H_{\ell}^{*}$-equivalent as well, since in this case $X_{1, \mathbb{C}}$ and $X_{2, \mathbb{C}}$ are homeomorphic. 
Theorem 1.3 (= Theorem 3.5) Let $K_{1}, K_{2}$ be subfields of $\mathbb{C}$, finitely generated over $\mathbb{Q}$, and let $X_{i}$ be a hyper-Kähler varietyover $K_{i}$, for $i=1,2$. Assume that $X_{1}$ and $X_{2}$ are $H_{\ell}^{*}$-equivalent, and that the Mumford-Tate conjecture holds for both of them. Let $\Gamma \subset \operatorname{Gal}\left(\bar{K}_{1} / K_{1}\right)$ be a subgroup, let $\epsilon: \Gamma \rightarrow \operatorname{Gal}\left(\bar{K}_{2} / K_{2}\right)$ be a homomorphism; $\Gamma$ acts on $H_{X_{1}, \ell}^{*}$ via its inclusion into $\operatorname{Gal}\left(\bar{K}_{1} / K_{1}\right)$ and on $H_{X_{2}, \ell}^{*}$ via $\epsilon$. Assume that there exists a $\Gamma$-equivariant isometry $f: H_{X_{1}, \ell}^{2} \rightarrow H_{X_{2}, \ell}^{2}$. Then, there exist a subgroup $\Gamma^{\prime} \subset \Gamma$ of finite index and an isomorphism $F: H_{X_{1}, \ell}^{*} \rightarrow H_{X_{2}, \ell}^{*}$ of graded algebras which is $\Gamma^{\prime}$-equivariant.

This result leads to similar conclusions for hyper-Kähler varieties over finite fields. The study of such varieties is still in its early stages: besides $\mathrm{K} 3$ surfaces, it is not clear how to define these objects (but a possible definition is proposed in [11]). Despite this, certain higher dimensional moduli spaces of sheaves on K3 surfaces play a key role in Charles's proof of the Tate conjecture for K3 surfaces [7] over finite fields; other examples of hyper-Kähler varieties over finite fields can be obtained from moduli spaces of sheaves on abelian surfaces [11]. All these varieties can be lifted to some hyper-Kähler variety in characteristic 0 . We will therefore consider smooth and projective varieties over finite fields which can be lifted to a hyper-Kähler variety in characteristic 0; see Sect. 3.2 for the precise meaning of this. The recent article [32] shows that this approach yields at least a good notion of varieties of $\mathrm{K} 3^{[n]}$-type.

Let $k$ be a finite field and let $\ell$ be a prime number invertible in $k$. For a smooth and projective variety $Z$ over $k$, we let $H_{Z, \ell}^{*}:=H_{\text {êt }}^{*}\left(Z_{\bar{k}}, \mathbb{Q}_{\ell}\right)$; if $Z$ can be lifted to a hyper-Kähler variety in characteristic 0 then the second cohomology group $H_{Z, \ell}^{2}$ inherits a non-degenerate $\mathbb{Q}_{\ell}$-valued symmetric bilinear pairing, see Remark 3.7.

Theorem 1.4 (= Theorem 3.8) Let $Z_{1}, Z_{2}$ be smooth projective varieties over $k$ such that there exist $H_{\ell}^{*}$-equivalent hyper-Kähler varieties $X_{1}, X_{2}$ in characteristic 0 which lift $Z_{1}$ and $Z_{2}$ respectively. Assume that the Mumford-Tate conjecture holds for both $X_{1}$ and $X_{2}$ and that there exists a $\mathrm{Gal}(\bar{k} / k)$-equivariant isometry $f: H_{Z_{1}, \ell}^{2} \rightarrow H_{Z_{2}, \ell}^{2}$. Then, there exist a finite field extension $k^{\prime}$ of $k$ and $a \mathrm{Gal}\left(\bar{k} / k^{\prime}\right)$-equivariant isomorphism of graded algebras $F: H_{Z_{1}, \ell}^{*} \rightarrow H_{Z_{2}, \ell}^{*}$

In particular $Z_{1, k^{\prime}}$ and $Z_{2, k^{\prime}}$ have the same zeta function. In the special case when $Z_{1}$ and $Z_{2}$ are moduli spaces of stable sheaves on $\mathrm{K} 3$ surfaces over $k$ the above statement has already been proven by Frei in [13] via a different method, which uses Markman's results from [19].

The structure of this article is reversed with respect to the order of the introduction. Namely, after reviewing in Sect. 2 the construction of the Looijenga-Lunts-Verbitsky (LLV) Lie algebra, we use the properties of this Lie algebra to prove Theorems 1.3 and 1.4 in Sect. 3. We then prove Theorem 1.1 and Corollary 1.2 in Sect. 4. These last results rely fundamentally on the defect groups of hyper-Kähler varieties introduced in [10] with Lie Fu and Ziyu Zhang. The proof of Theorem 1.1 also uses the fact that deformation equivalent hyper-Kähler varieties can be connected using polarized deformations, which is proven in Sect. 5.

\section{Notation and conventions}

By a hyper-Kähler variety over a field $K \subset \mathbb{C}$ we mean a smooth and projective variety $X$ over $K$ such that $X(\mathbb{C})$ is a complex hyper-Kähler manifold, i.e. it is simply connected and $H^{0}\left(X(\mathbb{C}), \Omega^{2}\right)$ is spanned by the cohomology class of a nowhere degenerate holomorphic 2 -form. If $X_{1}, X_{2}$ are hyper-Kähler varieties over subfields $K_{1}, K_{2} \subset \mathbb{C}$ respectively, we say 
that $X_{1}$ and $X_{2}$ are deformation equivalent if the complex manifolds $X_{1}(\mathbb{C})$ and $X_{2}(\mathbb{C})$ are deformation equivalent (in the analytic sense). For a smooth and projective variety $X$ over a subfield $K \subset \mathbb{C}$ we use the notation $H_{X}^{j}:=H^{j}(X(\mathbb{C}), \mathbb{Q})$ and $H_{X, \ell}^{j}:=H_{\text {èt }}^{j}\left(X_{\bar{K}}, \mathbb{Q}_{\ell}\right)$, where $\bar{K} \subset \mathbb{C}$ is the algebraic closure of $K$ in $\mathbb{C}$ and $\ell$ is a prime number.

\section{The LLV-Lie algebra}

In this section, we let $X$ be a complex hyper-Kähler variety of dimension $2 n$. We let $H_{X}^{*}:=\bigoplus_{j} H^{j}(X, \mathbb{Q})$; the second cohomology group $H_{X}^{2}$ is equipped with the BeauvilleBogomolov pairing $q$, a non-degenerate symmetric bilinear form, see [5, Théorème 5]. We define the Mukai extension of the quadratic space $\left(H_{X}^{2}, q\right)$ as the vector space $\tilde{H}_{X}^{2}:=\mathbb{Q} \cdot v \oplus H_{X}^{2} \oplus \mathbb{Q} \cdot w$, equipped with the pairing

$$
\tilde{q}\left((a v, b, c w),\left(a^{\prime} v, b^{\prime}, c^{\prime} w\right)\right)=q\left(b, b^{\prime}\right)-a c^{\prime}-a^{\prime} c .
$$

Given $x \in H_{X}^{2}$, let $L_{x}: H_{X}^{*} \rightarrow H_{X}^{*+2}$ be given by cup-product with $x$. We say that $x$ has the Lefschetz property if the maps $L_{x}^{k}: H_{X}^{2 n-k} \rightarrow H_{X}^{2 n+k}$ are isomorphisms for all $k>0$. Let $\theta$ denote the endomorphism of the cohomology which acts on $H_{X}^{j}$ as multiplication by $j-2 n$. It is well-known that the class $x$ has the Lefschetz property if and only if there exists $\Lambda_{x}: H_{X}^{*} \rightarrow H_{X}^{*-2}$ such that $\left(L_{x}, \theta, \Lambda_{x}\right)$ is an $\mathfrak{s l}_{2}$-triple, meaning that we have

$$
\left[\theta, L_{x}\right]=2 L_{x},\left[\theta, \Lambda_{x}\right]=-2 \Lambda_{x},\left[L_{x}, \Lambda_{x}\right]=\theta .
$$

If it exists, $\Lambda_{X}$ is uniquely determined. The subset of $x \in H_{X}^{2}$ with the Lefschetz property is Zariski open in $H_{X}^{2}$, and the first Chern class of an ample divisor on $X$ has the Lefschetz property by the Hard Lefschetz theorem.

Definition 2.1 The LLV-Lie algebra $\mathfrak{g}(X)$ of $X$ is the Lie subalgebra of $\mathfrak{g l}\left(H_{X}^{*}\right)$ generated by all $\mathfrak{s l}_{2}$-triples $\left(L_{x}, \theta, \Lambda_{x}\right)$ for $x \in H_{X}^{2}$ with the Lefschetz property. We let $\mathfrak{g}_{0}(X) \subset \mathfrak{g}(X)$ denote the centralizer of the semisimple element $\theta$.

In other words, $\mathfrak{g}_{0}(X)$ consists of those endomorphisms in $\mathfrak{g}(X)$ whose action on $H_{X}^{*}$ preserve the grading.

The LLV-Lie algebras of hyper-Kähler varieties have been fully described.

\section{Theorem $2.2[18,30]$}

(a) There exists a unique isomorphism of $\mathbb{Q}$-Lie algebras

$$
\varphi: \mathfrak{g}(X) \stackrel{\sim}{\longrightarrow} \mathfrak{s o}\left(\tilde{H}_{X}^{2}, \tilde{q}\right),
$$

such that:

- $\varphi(\theta)$ vanishes on $H_{X}^{2}, \varphi(\theta)(v)=-2 v$ and $\varphi(\theta)(w)=2 w$, and

- for any $x \in H_{X}^{2}$ with the Lefschetz property, we have $\varphi\left(L_{x}\right)(v)=x, \varphi\left(L_{x}\right)(w)=0$ and $\varphi\left(L_{x}\right)(y)=q(x, y) \cdot w$, for all $y \in H_{X}^{2}$.

(b) The isomorphism $\varphi$ restricts to an isomorphism

$$
\mathfrak{g}_{0}(X) \cong \mathfrak{s o}\left(H_{X}^{2}, q\right) \oplus \mathbb{Q} \cdot \varphi(\theta) ;
$$

the induced representation of $\mathfrak{s o}\left(H_{X}^{2}, q\right)$ on $H_{X}^{2}$ is the standard representation. 
For later use, we note the following functoriality property of the LLV-construction.

Lemma 2.3 Let $X_{1}$ and $X_{2}$ be hyper-Kähler varieties and let $F: H_{X_{1}}^{*} \rightarrow H_{X_{2}}^{*}$ be an isomorphism of graded algebras. Then the induced isomorphism $F_{*}: \operatorname{GL}\left(H_{X_{1}}^{*}\right) \rightarrow \operatorname{GL}\left(H_{X_{2}}^{*}\right)$ given by $A \mapsto F A F^{-1}$ restricts to an isomorphism $\mathfrak{g}\left(X_{1}\right) \rightarrow \mathfrak{g}\left(X_{2}\right)$.

Proof Let $x \in H_{X_{1}}^{2}$ be an element with the Lefschetz property, and consider the corresponding $\mathfrak{s l}_{2}$-triple $\left(L_{x}, \theta_{X_{1}}, \Lambda_{x}\right)$. Then $\left(F L_{x} F^{-1}, F \theta_{X_{1}} F^{-1}, F \Lambda_{x} F^{-1}\right)$ is again an $\mathfrak{s l}_{2}$-triple; moreover, since $F$ is an isomorphism of graded algebras it is immediate to check that $F L_{x} F^{-1}=L_{F(x)}$ and $F \theta_{X_{1}} F^{-1}=\theta_{X_{2}}$. Further, $F(x)$ has the Lefschetz property as well, and it follows that $F_{*}\left(\Lambda_{x}\right)=\Lambda_{F(x)}$. Since the Lie algebra $\mathfrak{g}\left(X_{1}\right)$ is generated by the $\mathfrak{s l}_{2}$-triples $\left(L_{x}, \theta_{X_{1}}, \Lambda_{x}\right)$ as above, this concludes the proof.

Remark 2.4 Together with Theorem 2.2, Lemma 2.3 implies that any linear isomorphism $f: H_{X_{1}}^{2} \rightarrow H_{X_{2}}^{2}$ which extends to an isomorphism $H_{X_{1}}^{*} \rightarrow H_{X_{2}}^{*}$ of graded algebras induces an isomorphism $f_{*}: \mathfrak{s o}\left(H_{X_{1}}^{2}\right) \rightarrow \mathfrak{s o}\left(H_{X_{2}}^{2}\right)$. This fact can be seen as a consequence of Fujiki's relation, which gives positive rational constants $\lambda_{i}$, for $i=1,2$, such that $q(\alpha, \alpha)^{n}=$ $\lambda_{i} \int_{X_{i}} \alpha^{2 n}$ for any $\alpha \in H_{X_{i}}^{2}$, see [15, Sect. 1.11].

\subsection{The integrated representation}

We let $\mathrm{G}(X)$ be the semisimple simply connected algebraic group with Lie algebra $\mathfrak{g}(X)$, and let $\mathrm{G}_{0}(X) \subset \mathrm{G}(X)$ be the unique connected subgroup with Lie algebra $\mathfrak{g}_{0}(X)$. By Theorem 2.2 we have an isomorphism

$$
\tilde{\varphi}: \mathrm{G}(X) \stackrel{\sim}{\longrightarrow} \operatorname{Spin}\left(\tilde{H}_{X}^{2}, \tilde{q}\right) .
$$

Let $U:=\langle v, w\rangle$, equipped with the restriction of $\tilde{q}$. Since $\tilde{H}_{X}^{2}=H_{X}^{2} \oplus U$, we can view $\operatorname{Spin}\left(H_{X}^{2}, q\right)$ and $\operatorname{Spin}(U)$ as algebraic subgroups of $\operatorname{Spin}\left(\tilde{H}_{X}^{2}, \tilde{q}\right)$. We have $\operatorname{Spin}(U) \cong \mathbb{G}_{m}$, and the Lie algebra of $\operatorname{Spin}(U) \subset \operatorname{Spin}\left(\tilde{H}_{X}^{2}, \tilde{q}\right)$ is $\mathbb{Q} \cdot \varphi(\theta)$. Moreover, $\operatorname{Spin}\left(H_{X}^{2}, q\right) \cap$ $\operatorname{Spin}(U)=\{ \pm 1\}$. We conclude that $\tilde{\varphi}$ restricts to an isomorphism

$$
\tilde{\varphi}: \mathrm{G}_{0}(X) \stackrel{\sim}{\longrightarrow} \operatorname{CSpin}\left(H_{X}^{2}, q\right)=\operatorname{Spin}\left(H_{X}^{2}, q\right) \cdot \operatorname{Spin}(U) .
$$

The above assertions are checked as follows. With respect to the basis $\left\{v,-\frac{w}{2}\right\}$, the matrix of $\tilde{q}_{\left.\right|_{U}}$ is $\left(\begin{array}{cc}0 & 1 / 2 \\ 1 / 2 & 0\end{array}\right)$. Let $\mathrm{Cl}(U)$ be the Clifford algebra on $U$. Then $\mathrm{Cl}(U)$ is identified with the algebra of 2 by 2 matrices with coefficients in $\mathbb{Q}$; an isomorphism is given by

$$
v \mapsto\left(\begin{array}{ll}
0 & 0 \\
1 & 0
\end{array}\right),-\frac{w}{2} \mapsto\left(\begin{array}{ll}
0 & 1 \\
0 & 0
\end{array}\right) \text {. }
$$

The even Clifford algebra $\mathrm{Cl}^{+}(U)$ consists of the diagonal matrices, while $\mathrm{Cl}^{-}(U)$ consists of those matrices with 0 on the diagonal. The spinor norm $\mathrm{Cl}(U)^{\times} \rightarrow \mathbb{Q}^{\times}$is the determinant. Therefore $\operatorname{Spin}(U) \cong \mathbb{G}_{m}$ is the standard maximal torus of $\mathrm{SL}_{2}$. The adjoint action of $\operatorname{Spin}(U)$ on $\tilde{H}_{X}^{2}$ is trivial on the summand $H_{X}^{2}$, and we have

$$
\left(\begin{array}{cc}
\lambda & 0 \\
0 & \lambda^{-1}
\end{array}\right) v\left(\begin{array}{cc}
\lambda^{-1} & 0 \\
0 & \lambda
\end{array}\right)=\lambda^{-2} v,\left(\begin{array}{cc}
\lambda & 0 \\
0 & \lambda^{-1}
\end{array}\right) w\left(\begin{array}{cc}
\lambda^{-1} & 0 \\
0 & \lambda
\end{array}\right)=\lambda^{2} w .
$$

This implies that the subgroup $\operatorname{Spin}(U) \subset \operatorname{Spin}\left(\tilde{H}_{X}^{2}, \tilde{q}\right)$ corresponds to the Lie subalgebra $\mathbb{Q} \cdot \theta$ of $\mathfrak{s o}\left(\tilde{H}_{X}^{2}, \tilde{q}\right)$. Finally, since $\operatorname{Cl}\left(\tilde{H}_{X}^{2}, \tilde{q}\right)=\mathrm{Cl}\left(H_{X}^{2}, q\right) \otimes \mathrm{Cl}\left(U, \tilde{q}_{\left.\right|_{U}}\right)$, it is clear that we have $\operatorname{Spin}\left(H_{X}^{2}, q\right) \cap \operatorname{Spin}(U)=\{ \pm 1\}$. 
The action of $\mathfrak{g}(X)$ on $H_{X}^{*}$ integrates to a representation $\tilde{\rho}: \mathrm{G}(X) \rightarrow \mathrm{GL}\left(H_{X}^{*}\right)$, which restricts to

$$
\tilde{\rho}_{0}: \mathrm{G}_{0}(X) \rightarrow \prod_{j} \mathrm{GL}\left(H_{X}^{j}\right)
$$

We denote by $\tilde{\rho}_{0}^{(2)}: \mathrm{G}_{0}(X) \rightarrow \mathrm{GL}\left(H_{X}^{2}\right)$ its degree 2 component.

In what follows, we identify $\mathrm{G}(X)$ with $\operatorname{Spin}\left(\tilde{H}_{X}^{2}, \tilde{q}\right)$ and $\mathrm{G}_{0}(X)$ with $\operatorname{CSpin}\left(H_{X}^{2}, q\right)$ via $\tilde{\varphi}$. If there is no risk of confusion, we $\operatorname{simply}$ write $\mathrm{CSpin}\left(H_{X}^{2}\right)$ for $\mathrm{CSpin}\left(H_{X}^{2}, q\right)$, and similarly for other groups.

Remark 2.5 Consider the element $-1 \in \operatorname{CSpin}\left(H_{X}^{2}\right) \subset \operatorname{Spin}\left(\tilde{H}_{X}^{2}\right)$. It has been shown by Verbitsky in [29, Sect. 8] that for all $j \geq 0$ and any $v \in H_{X}^{j}$ we have $\tilde{\rho}(-1)(v)=(-1)^{j}$. Combining this with Theorem 2.2, it follows that $\tilde{\rho}$ is faithful if $X$ has non-trivial cohomology in some odd degree, and that $\tilde{\rho}$ has kernel $\{ \pm 1\}$ otherwise.

The connected center of the algebraic group $\operatorname{CSpin}\left(H_{X}^{2}\right)$ is the subgroup $\mathbb{G}_{m}$ of invertible scalars in the Clifford algebra, and we have short exact sequences of algebraic groups

$$
1 \longrightarrow \mathbb{G}_{m} \longrightarrow \operatorname{CSpin}\left(H_{X}^{2}\right) \stackrel{\pi}{\longrightarrow} \mathrm{SO}\left(H_{X}^{2}\right) \longrightarrow 1
$$

and

$$
1 \longrightarrow \operatorname{Spin}\left(H_{X}^{2}\right) \longrightarrow \mathrm{CSpin}\left(H_{X}^{2}\right) \stackrel{\mathrm{Nm}}{\longrightarrow} \mathbb{G}_{m} \longrightarrow 1
$$

such that for all $z \in \mathbb{G}_{m} \subset \operatorname{CSpin}\left(H_{X}^{2}\right)$ we have $\operatorname{Nm}(z)=z^{2}$.

In addition to the representation $\tilde{\rho}_{0}$, we will consider a second, twisted, action of $\mathrm{CSpin}\left(H_{X}^{2}\right)$ on $H_{X}^{*}$, which we will refer to as the $R$-action. It is defined via the homomorphism

$$
R: \operatorname{CSpin}\left(H_{X}^{2}\right) \rightarrow \prod_{j} \mathrm{GL}\left(H_{X}^{j}\right)
$$

given by $R(g)=\mathrm{Nm}(g)^{n} \cdot \tilde{\rho}_{0}(g)$.

Lemma 2.6 The R-action on $H_{X}^{*}$ is an action by graded algebra automorphisms.

Proof It has been shown in [18, (4.4)] that the semisimple part $\mathfrak{s o}\left(H_{X}^{2}\right)$ of the Lie algebra of CSpin $\left(H_{X}^{2}\right)$ acts on the cohomology algebra via derivations; it follows that the subgroup $\operatorname{Spin}\left(H_{X}^{2}\right)$ acts on $H_{X}^{*}$ by graded algebra isomorphisms. Moreover for any $z \in \mathbb{G}_{m}$ and $y \in$ $H_{X}^{j}$ we have $\tilde{\rho}_{0}(z)(y)=z^{j-2 n} \cdot y$. Thus the factor $\operatorname{Nm}(z)^{n}=z^{2 n}$ ensures that also the action of $\mathbb{G}_{m} \subset \mathrm{CSpin}\left(H_{X}^{2}\right)$ on $H_{X}^{*}$ is by algebra automorphisms. As $C \operatorname{Spin}\left(H_{X}^{2}\right)=\mathbb{G}_{m} \cdot \operatorname{Spin}\left(H_{X}^{2}\right)$, this concludes the proof.

Remark 2.7 The homomorphism

$$
(\mathrm{Nm}, \pi): \operatorname{CSpin}\left(H_{X}^{2}\right) \rightarrow \mathbb{G}_{m} \times \mathrm{SO}\left(H_{X}^{2}\right)
$$

is surjective with kernel $\{ \pm 1\}$. By Remark 2.5, the $R$-action on the even cohomology factors through $(\mathrm{Nm}, \pi)$. If $g \in \mathrm{CSpin}\left(H_{X}^{2}\right)$, then the degree 2 component $R^{(2)}(g)$ of $R(g)$ equals $\mathrm{Nm}(g) \cdot \pi(g)$, while for $\tilde{\rho}_{0}(g)$ we have $\tilde{\rho}_{0}^{(2)}(g)=\mathrm{Nm}(g)^{1-n} \cdot \pi(g)$.

The combination of this observation with Theorem 2.2 implies that the natural homomorphism $R\left(\mathrm{G}_{0}(X)\right) \rightarrow R^{(2)}\left(\mathrm{G}_{0}(X)\right)$ is an isomorphism if the odd cohomology of $X$ vanishes, and it has kernel $\{ \pm 1\}$ otherwise. 


\subsection{Hodge structures of hyper-Kähler varieties}

Given a $\mathbb{Q}$-Hodge structure $V$, we let $\mathrm{MT}(V)$ denote its Mumford-Tate group. As a consequence of a result of Verbitsky [30], it is known that the LLV-Lie algebra of a hyper-Kähler variety controls the Hodge structure on its cohomology. We summarize this result as follows, see [10, Lemma 6.7].

Theorem 2.8 The Mumford-Tate group $\mathrm{MT}\left(H_{X}^{*}\right)$ is contained in the image of the representation $R: \operatorname{CSpin}\left(H_{X}^{2}\right) \rightarrow \prod_{j} \operatorname{GL}\left(H_{X}^{j}\right)$.

We have the following consequence.

Proposition 2.9 Let $X_{1}$ and $X_{2}$ be complex hyper-Kähler varieties and let $F: H_{X_{1}}^{*} \rightarrow H_{X_{2}}^{*}$ be an isomorphism of graded algebras. Assume that the degree 2 component $F^{(2)}: H_{X_{1}}^{2} \rightarrow H_{X_{2}}^{2}$ is an isomorphism of Hodge structures. Then $F$ is an isomorphism of Hodge structures.

Proof Let $\mathbb{S}:=\operatorname{Res}_{\mathbb{R}}^{\mathbb{C}}\left(\mathbb{G}_{m}\right)$ be the Deligne torus. The total Hodge structure on $H_{X_{i}}^{*}$ corresponds to a real representation

$$
h_{X_{i}}: \mathbb{S} \rightarrow \prod_{j} \mathrm{GL}\left(H_{X_{i}}^{j}\right) \otimes \mathbb{R}
$$

by definition, $h_{X_{i}}$ factors through $\operatorname{MT}\left(H_{X_{i}}^{*}\right)(\mathbb{R})$. By Theorem 2.8 the group $\operatorname{MT}\left(H_{X_{i}}^{*}\right)$ is contained in the image of the representation $R: \mathrm{G}_{0}\left(X_{i}\right) \rightarrow \prod_{j} \mathrm{GL}\left(H_{X_{i}}^{j}\right)$. By Lemma 2.3, the induced isomorphism $F_{*}: \operatorname{GL}\left(H_{X_{1}}^{*}\right) \rightarrow \operatorname{GL}\left(H_{X_{2}}^{*}\right)$ restricts to an isomorphism $\mathfrak{g}\left(X_{1}\right) \cong$ $\mathfrak{g}\left(X_{2}\right)$; since moreover $F$ preserves the cohomological grading, $F_{*}$ restricts to an isomorphism $R\left(\mathrm{G}_{0}\left(X_{1}\right)\right) \cong R\left(\mathrm{G}_{0}\left(X_{2}\right)\right)$.

We have to prove that the diagram

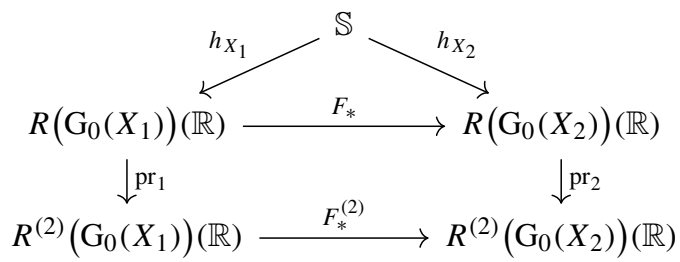

is commutative. By Remark 2.7, the morphism $\mathrm{pr}_{2}: R\left(\mathrm{G}_{0}\left(X_{2}\right)\right) \rightarrow R^{(2)}\left(\mathrm{G}_{0}\left(X_{2}\right)\right)$ is either an isomorphism or a central isogeny of degree 2; let $C$ be the kernel. Since $F^{(2)}$ is an isomorphism of Hodge structures, we have $F_{*}^{(2)} \circ \mathrm{pr}_{1} \circ h_{X_{1}}=\mathrm{pr}_{2} \circ h_{X_{2}}$. Hence, there is a morphism $\xi: \mathbb{S} \rightarrow C$ such that $F_{*} \circ h_{X_{1}}=\xi \cdot h_{X_{2}}$. But $\mathbb{S}$ is connected and $C$ is finite, so $\xi$ is trivial and $F$ is an isomorphism of Hodge structures.

Remark 2.10 In fact Theorem 2.8 (and hence also the Proposition) holds more generally for complex hyper-Kähler manifolds which are not necessarily projective.

\section{Galois representations}

Throughout this section, $\ell$ will denote a fixed prime number. Let $K \subset \mathbb{C}$ be a field that is finitely generated over $\mathbb{Q}$, and let $\bar{K}$ be the algebraic closure of $K$ in $\mathbb{C}$. By a hyper-Kähler 
variety over $K$ we mean a smooth projective variety $X$ over $K$ such that the base change $X_{\mathbb{C}}$ is a complex hyper-Kähler variety. For all integers $j$ and $m$, we have canonical comparison isomorphisms

$$
H_{\mathrm{e} t}^{j}\left(X_{\bar{K}}, \mathbb{Q}_{\ell}\right)(m) \cong H^{j}\left(X_{\mathbb{C}}, \mathbb{Q}\right)(m) \otimes_{\mathbb{Q}} \mathbb{Q}_{\ell} .
$$

We let $H_{X}^{*}:=\bigoplus_{j} H^{j}\left(X_{\mathbb{C}}, \mathbb{Q}\right)$ and adopt the notation from the previous section. We define $H_{X, \ell}^{*}:=\bigoplus_{j} H_{\text {êt }}^{j}\left(X_{\bar{K}}, \mathbb{Q}_{\ell}\right)$. We will identify $H_{X, \ell}^{j}$ with $H_{X}^{j} \otimes_{\mathbb{Q}} \mathbb{Q}_{\ell}$; the Beauville-Bogomolov form extends to a non-degenerate $\mathbb{Q}_{\ell}$-valued bilinear pairing on $H_{X, \ell}^{2}$.

There is a continuous representations

$$
\sigma_{X}: \operatorname{Gal}(\bar{K} / K) \rightarrow \prod_{j} \operatorname{GL}\left(H_{X, \ell}^{j}\right) .
$$

We denote by $G\left(H_{X, \ell}^{*}\right) \subset \operatorname{GL}\left(H_{X, \ell}^{*}\right)$ the Zariski closure of the image of $\sigma_{X}$. The group $G\left(H_{X, \ell}^{*}\right)$ is not connected in general. The identity component $G\left(H_{X, \ell}^{*}\right)^{0}$ of this group remains invariant under finitely generated field extensions of $K$. After replacing $K$ with a finite field extension $\hat{K} / K$, the group $G\left(H_{X, \ell}^{*}\right)$ becomes connected, see [22, Remarks 2.2.2].

Let $K \subset \bar{K} \subset \mathbb{C}$ be as above. The Mumford-Tate conjecture aims to compare the Hodge structure on $H_{X}^{*}$ with the Galois representation $\sigma_{X}$ on $H_{X, \ell}^{*}$.

Conjecture 3.1 (Mumford-Tate conjecture) For any smooth and projective variety $X$ over $K$, the comparison isomorphism $H_{X, \ell}^{*} \cong H_{X}^{*} \otimes \mathbb{Q}_{\ell}$ induces an isomorphism of connected algebraic groups

$$
G\left(H_{X, \ell}^{*}\right)^{0} \cong \operatorname{MT}\left(H_{X}^{*}\right) \otimes_{\mathbb{Q}} \mathbb{Q}_{\ell}
$$

Let us note that the version of the Mumford-Tate conjecture given here is stronger than the one which says that under the comparison isomorphism $H_{X, \ell}^{j} \cong H_{X}^{j} \otimes_{\mathbb{Q}} \mathbb{Q}_{\ell}$ the group $G\left(H_{X, \ell}^{j}\right)^{0}$ is identified with $\operatorname{MT}\left(H_{X}^{j}\right) \otimes \mathbb{Q}_{\ell}$ for all $j$.

The Mumford-Tate conjecture does not depend on the base field, and it may even be formulated for varieties over the complex numbers, see [23, Sect. 1.6].

At present, four deformation types of complex hyper-Kähler varieties are known besides $\mathrm{K} 3$ surfaces, commonly referred to as the deformation types $\mathrm{K} 3^{[n]}$ and $\mathrm{Kum}^{n}$ [5], for all $n \geq 2$, and O'Grady's deformation types OG10 [24] and OG6 [25]. Together with Lie Fu and Ziyu Zhang, we have proven the Mumford-Tate conjecture for all hyper-Kähler varieties of one of these types (the case of $\mathrm{K} 3^{[n]}$-type varieties was dealt with in [12]).

Theorem 3.2 [10, Theorem 1.18] Let $X$ be a hyper-Kähler variety over $K$. Assume that $X$ is deformation equivalent to one of the known examples, that is, $X_{\mathbb{C}}$ is of deformation type $\mathrm{K}^{[n]}{ }^{\left[n \mathrm{Kum}^{n}\right.}$, OG10 or OG6. Then the Mumford-Tate Conjecture 3.1 holds for X.

The Mumford-Tate conjecture in degree 2 is known for arbitrary hyper-Kähler varieties $X$ with $b_{2}(X)>3$ by [1]; see also [23].

\subsection{Galois representations of hyper-Kähler varieties}

Let now $K_{1}, K_{2}$ be subfields of $\mathbb{C}$, finitely generated over $\mathbb{Q}$, and consider hyper-Kähler varieties $X_{1}, X_{2}$ over $K_{1}$ and $K_{2}$ respectively. 
Definition 3.3 We say that $X_{1}$ and $X_{2}$ are $H_{\ell}^{*}$-equivalent if there exists an isomorphism of graded algebras $H_{X_{1}, \ell}^{*} \cong H_{X_{2}, \ell}^{*}$ which is an isometry in degree 2 .

Note that if $X_{1}$ and $X_{2}$ are deformation equivalent then they are $H_{\ell}^{*}$-equivalent, since in this case the manifolds $X_{1, \mathbb{C}}$ and $X_{2, \mathbb{C}}$ are homeomorphic, and both the graded algebra $H_{X_{i}}^{*}$ and the Beauville-Bogomolov form on $H_{X_{i}}^{2}$ only depend on the topology of the complex manifold $X_{i, \mathbb{C}}$.

Proposition 3.4 Let $X_{1}$ and $X_{2}$ be $H_{\ell}^{*}$-equivalent hyper-Kähler varieties over $K_{1}$ and $K_{2}$ respectively. Assume given a subgroup $\Gamma \subset \operatorname{Gal}\left(\bar{K}_{1} / K_{1}\right)$ and a homomorphism $\epsilon: \Gamma \rightarrow$ $\operatorname{Gal}\left(\bar{K}_{2} / K_{2}\right)$; we let $\Gamma$ act on $H_{X_{1}, \ell}^{*}$ via $\sigma_{X_{1}}$ and on $H_{X_{2}, \ell}^{*}$ via $\epsilon \circ \sigma_{X_{2}}$. If there exists an isometry $f: H_{X_{1}, \ell}^{2} \rightarrow H_{X_{2}, \ell}^{2}$ which is $\Gamma$-equivariant, then there exists an isomorphism $F: H_{X_{1}, \ell}^{*} \rightarrow H_{X_{2}, \ell}^{*}$ of graded algebras whose degree 2 component is again $\Gamma$-equivariant.

Proof Since $X_{1}$ and $X_{2}$ are $H_{\ell}^{*}$-equivalent, there exists an isomorphism of graded algebras $\Psi: H_{X_{1}, \ell}^{*} \rightarrow H_{X_{2}, \ell}^{*}$ which is an isometry in degree 2 ; let $\psi=\Psi^{(2)}$ be its component in degree 2 . We have $\psi^{-1} \circ f \in \mathrm{O}\left(H_{X_{1}}^{2}\right)\left(\mathbb{Q}_{\ell}\right)$. We may assume this isometry has determinant 1 , for if it has determinant -1 we can choose an ample line bundle on $X_{1}$ with first Chern class $e \in H_{X_{1}, \ell}^{2}$ and replace $f$ with the isometry given by $e \mapsto-f(e)$ and $v \mapsto f(v)$ for any $v \in\langle e\rangle^{\perp}$, which is again $\Gamma$-equivariant.

The morphism $\pi: \operatorname{CSpin}\left(H_{X_{1}}^{2}\right) \rightarrow \operatorname{SO}\left(H_{X_{1}}^{2}\right)$ is surjective on $\mathbb{Q}_{\ell}$-points. Indeed, by Hilbert's theorem 90 [26, Chapter X, Sect. 1], the short exact sequence

$$
1 \rightarrow \mathbb{G}_{m} \rightarrow \operatorname{CSpin}\left(H_{X_{1}}^{2}\right) \rightarrow \operatorname{SO}\left(H_{X_{1}}^{2}\right) \rightarrow 1
$$

yields a short exact sequence

$$
1 \rightarrow \mathbb{Q}_{\ell}^{\times} \rightarrow \operatorname{CSpin}\left(H_{X_{1}}^{2}\right)\left(\mathbb{Q}_{\ell}\right) \rightarrow \operatorname{SO}\left(H_{X_{1}}^{2}\right)\left(\mathbb{Q}_{\ell}\right) \rightarrow 1
$$

Therefore, there exists $g \in \operatorname{CSpin}\left(H_{X_{1}}^{2}\right)\left(\mathbb{Q}_{\ell}\right)$ such that $\pi(g)=\psi^{-1} \circ f$. By Lemma 2.6, $R(g)$ is a graded algebra automorphism of $H_{X_{1}, \ell}^{*}$. It follows that $F:=\Psi \circ R(g): H_{X_{1}, \ell}^{*} \rightarrow H_{X_{2}, \ell}^{*}$ is an isomorphism of graded algebras. By Remark 2.7, the degree 2 component $F^{(2)}$ of $F$ is $\mathrm{Nm}(g) \cdot f$, and hence it is $\Gamma$-equivariant.

We can now prove Theorem 1.3, whose statement is recalled below.

Theorem 3.5 Let $K_{1}, K_{2}$ be subfields of $\mathbb{C}$, finitely generated over $\mathbb{Q}$, and let $X_{i}$ be a hyperKähler variety over $K_{i}$, for $i=1,2$. Assume that $X_{1}$ and $X_{2}$ are $H_{\ell}^{*}$-equivalent and that the Mumford-Tate conjecture holds for both of them. Let $\Gamma \subset \operatorname{Gal}\left(\bar{K}_{1} / K_{1}\right)$ be a subgroup, let $\epsilon: \Gamma \rightarrow \operatorname{Gal}\left(\bar{K}_{2} / K_{2}\right)$ be a homomorphism and let $f: H_{X_{1}, \ell}^{2} \rightarrow H_{X_{2}, \ell}^{2}$ be a $\Gamma$-equivariant isometry. Then, there exist a subgroup $\Gamma^{\prime} \subset \Gamma$ of finite index and a $\Gamma^{\prime}$-equivariant isomorphism of graded algebras $F: H_{X_{1}, \ell}^{*} \rightarrow H_{X_{2}, \ell}^{*}$.

Proof Replacing $K_{i}$ by a finite field extension if necessary, we may assume that $G\left(H_{X_{i}, \ell}^{*}\right)$ is connected for $i=1,2$. Since the Mumford-Tate conjecture holds for $X_{i}$, by Theorem 2.8 the representation $\sigma_{X_{i}}: \operatorname{Gal}\left(\bar{K}_{i} / K_{i}\right) \rightarrow \operatorname{GL}\left(H_{X_{i}, \ell}^{*}\right)$ factors through the $\mathbb{Q}_{\ell}$-points of the image of the LLV-representation $R: \mathrm{G}_{0}\left(X_{i}\right) \rightarrow \prod_{j} \mathrm{GL}\left(H_{X_{i}}^{j}\right)$.

By Proposition 3.4 there exists an isomorphism $F: H_{X_{1}, \ell}^{*} \rightarrow H_{X_{2}, \ell}^{*}$ of graded algebras whose degree 2 component $F^{(2)}$ is $\Gamma$-equivariant. Now the argument is the same as in the proof of Proposition 2.9. We consider the isomorphism $F_{*}: \operatorname{GL}\left(H_{X_{1}, \ell}^{*}\right) \rightarrow \operatorname{GL}\left(H_{X_{2}, \ell}^{*}\right)$ 
given by $A \mapsto F A F^{-1}$, and the analogous isomorphism $F_{*}^{(2)}: \operatorname{GL}\left(H_{X_{1}, \ell}^{2}\right) \rightarrow \operatorname{GL}\left(H_{X_{2}, \ell}^{2}\right)$. By Lemma 2.3 the isomorphism $F_{*}$ restricts to an isomorphism $R\left(\mathrm{G}_{0}\left(X_{1}\right)\right)\left(\mathbb{Q}_{\ell}\right) \cong$ $R\left(\mathrm{G}_{0}\left(X_{2}\right)\right)\left(\mathbb{Q}_{\ell}\right)$.

We consider the diagram

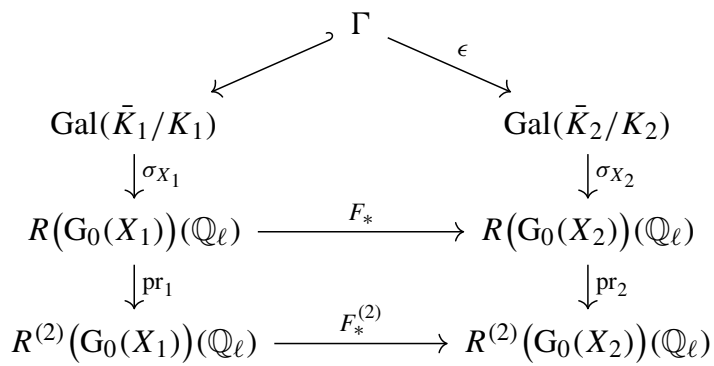

We have to show that, up to replacing $\Gamma$ by a subgroup of finite index, this diagram commutes.

Since $F^{(2)}$ is $\Gamma$-equivariant by assumption, we have $F_{*}^{(2)} \circ \mathrm{pr}_{1} \circ \sigma_{X_{1}}=\mathrm{pr}_{2} \circ \sigma_{X_{2}} \circ \epsilon$. By Remark 2.7, the homomorphism $\mathrm{pr}_{2}: R\left(\mathrm{G}_{0}\left(X_{2}\right)\right) \rightarrow R^{(2)}\left(\mathrm{G}_{0}\left(X_{2}\right)\right)$ is either an isomorphism or a central isogeny of degree 2 ; let $C$ be its kernel. Then there exists a homomorphism $\chi: \Gamma \rightarrow C\left(\mathbb{Q}_{\ell}\right)$ such that $F_{*} \circ \sigma_{X_{1}}(\gamma)=\chi(\gamma) \cdot \sigma_{X_{2}}(\gamma)$ for any $\gamma \in \Gamma$. The kernel $\Gamma^{\prime} \subset \Gamma$ of $\chi$ is a subgroup of finite index such that $F$ is $\Gamma^{\prime}$-equivariant.

Remark 3.6 Note that in the above proof we have only used that $G\left(H_{X_{i}, \ell}^{*}\right)^{0} \subset \operatorname{MT}\left(H_{X_{i}}^{*}\right)\left(\mathbb{Q}_{\ell}\right)$, so we only need one of the two inclusions predicted by the Mumford-Tate conjecture.

\subsection{An application to hyper-Kähler varieties over finite fields}

We apply Theorem 3.5 to the study of Galois representations on the cohomology of hyperKähler varieties over finite fields. We will consider the following situation. Let $k$ be a finite field, and let $Z_{1}$ and $Z_{2}$ be smooth projective varieties over $k$. We assume that there exist hyper-Kähler varieties $X_{1}$ and $X_{2}$ over fields of characteristic 0 which lift $Z_{1}$ and $Z_{2}$. More precisely, we assume that there exist:

- normal integral domains $R_{i} \subset \mathbb{C}$ essentially of finite type over $\mathbb{Z}$ (i.e. the localization of a finite type algebra over $\mathbb{Z}$ ) with fraction fields $K_{i}$ of characteristic 0 ;

- smooth and projective morphisms $\mathfrak{X}_{i} \rightarrow \operatorname{Spec}\left(R_{i}\right)$ whose generic fibres $X_{i}$ are hyperKähler;

- homomorphisms $R_{i} \rightarrow k$ together with isomorphisms $\mathfrak{X}_{i} \otimes_{R_{i}} k \cong Z_{i}$ of $k$-schemes.

We let $\ell$ be a prime number invertible in $k$ and consider $H_{Z_{i}, \ell}^{*}=\bigoplus_{j} H_{\text {ét }}^{j}\left(Z_{i, \bar{k}}, \mathbb{Q}_{\ell}\right)$. By the smooth and proper base-change theorems we have an isomorphism $H_{X_{i}, \ell}^{*} \cong H_{Z_{i}, \ell}^{*}$ of graded algebras. Via this isomorphism, the Beauville-Bogomolov form induces a non-degenerate symmetric bilinear form on $H_{Z_{i}, \ell}^{2}$.

Remark 3.7 A priori, the bilinear form that we obtain on $H_{Z_{i}, \ell}^{2}$ depends on the choices of $R_{i}$ and $\mathfrak{X}_{i}$. However, by [16, Sect. 4], the formula

$$
\alpha \mapsto \int_{X_{i}} \alpha^{2} \wedge \sqrt{\operatorname{td}\left(X_{i}\right)}
$$


defines a non-degenerate quadratic form on $H_{X_{i}}^{2}$ which is a non-zero multiple of the Beauville-Bogomolov form. The induced form on $H_{Z_{i}, \ell}^{2}$ is $\alpha \mapsto \int_{Z_{i}} \alpha^{2} \wedge \sqrt{\operatorname{td}\left(Z_{i}\right)}$, and it is thus independent from the choices of $R_{i}$ and $\mathfrak{X}_{i}$.

We now prove Theorem 1.4.

Theorem 3.8 With notations and assumptions as above, assume that $X_{1}$ and $X_{2}$ are $H_{\ell}^{*}$ equivalent, and that the Mumford-Tate conjecture holds for both of them. Let $f: H_{Z_{1}, \ell}^{2} \rightarrow$ $H_{Z_{2}, \ell}^{2}$ be a $\mathrm{Gal}(\bar{k} / k)$-equivariant isometry. Then, there exist a finite field extension $k^{\prime}$ of $k$ and $a \mathrm{Gal}\left(\bar{k} / k^{\prime}\right)$-equivariant isomorphism of graded algebras $F: H_{Z_{1}, \ell}^{*} \rightarrow H_{Z_{2}, \ell}^{*}$.

Proof Let $|k|=p^{r}$, and let $\operatorname{Fr}_{k} \in \operatorname{Gal}(\bar{k} / k)$ be the Frobenius automorphism. With notations as in above, let $\mathfrak{m}_{i} \subset R_{i}$ be the kernel of $R_{i} \rightarrow k$; let $\left|R_{i} / \mathfrak{m}_{i}\right|=p^{r / a_{i}}$ and denote by $\phi_{i} \in \operatorname{Gal}\left(\bar{K}_{i} / K_{i}\right)$ a Frobenius element at $\mathfrak{m}_{i}$, for $i=1,2$.

By construction, we have isomorphisms $\left\langle\phi_{i}^{a_{i}}\right\rangle=\left\langle\operatorname{Fr}_{k}\right\rangle$ (both isomorphic to $\mathbb{Z}$ ) such that the action of $\phi^{a_{i}}$ on $H_{Z_{i}, \ell}^{*}$ via the base-change isomorphism $H_{X_{i}, \ell}^{*} \cong H_{Z_{i}, \ell}^{*}$ corresponds to that of $\mathrm{Fr}_{k}$.

Let now $\Gamma=\left\langle\phi_{1}^{a_{1}}\right\rangle \subset \operatorname{Gal}\left(\bar{K}_{1} / K\right)$ and let $\epsilon: \Gamma \rightarrow \operatorname{Gal}\left(\bar{K}_{2} / K_{2}\right)$ be the homomorphism such that $\phi_{1}^{a_{1}} \mapsto \phi_{2}^{a_{2}}$. By Theorem 3.5, there exists an integer $m$ and an isomorphism $H_{Z_{1}, \ell}^{*} \rightarrow H_{Z_{2}, \ell}^{*}$ of graded algebras which is $\mathrm{Fr}_{k}^{m}$-equivariant.

\section{Motives}

Let $K \subset \mathbb{C}$ be a subfield. We will work with the category of motives over $K$ introduced by André in [2], which we denote by $\mathrm{AM}_{K}$. It is a neutral tannakian semisimple abelian category, with functors

$$
\operatorname{SmProj}_{K}^{\text {op }} \stackrel{\mathcal{H}^{j}}{\rightarrow} \mathrm{AM}_{K} \stackrel{r}{\rightarrow} \mathrm{HS}_{\mathbb{Q}}^{\text {pol }}
$$

such that $r \circ \mathcal{H}^{j}$ is the functor $H^{j}$ which associates to a smooth and projective variety $X$ the rational Hodge structure $H_{X}^{j}$. We denote by $\mathcal{H}_{X}^{*}:=\bigoplus_{j} \mathcal{H}_{X}^{j}$ the motive of the smooth and projective variety $X$.

The composition of the realization functor $r$ with the forgetful functor to $\mathbb{Q}$-vector spaces is a fibre functor on $\mathrm{AM}_{K}$; the tensor automorphisms of this functor form a pro-reductive group $\mathcal{G}_{\text {mot }}\left(\mathrm{AM}_{K}\right)$ over $\mathbb{Q}$, and $\mathrm{AM}_{K}$ is equivalent to the category of finite dimensional $\mathbb{Q}$-representations of $\mathcal{G}_{\text {mot }}\left(\mathrm{AM}_{K}\right)$.

We denote by $\langle\mathcal{M}\rangle$ the tannakian subcategory of $\mathrm{AM}_{K}$ generated by a motive $\mathcal{M}$. Via restriction to $\langle\mathcal{M}\rangle$ of the fibre functor described above, we obtain a reductive algebraic group $\mathrm{G}_{\text {mot }}(\mathcal{M}) \subset \mathrm{GL}(r(\mathcal{M}))$ whose category of representations is equivalent to $\langle\mathcal{M}\rangle$; we have a canonical surjective homomorphism $\mathcal{G}_{\text {mot }}\left(\mathrm{AM}_{K}\right) \rightarrow \mathrm{G}_{\text {mot }}(\mathcal{M})$. If $\mathcal{M}, \mathcal{N} \in \mathrm{AM}_{K}$, a linear isomorphism $f: r(\mathcal{M}) \rightarrow r(\mathcal{N})$ between their realizations comes from an isomorphism of motives if and only if it is $\mathcal{G}_{\text {mot }}\left(\mathrm{AM}_{K}\right)$-equivariant.

Remark 4.1 If $K^{\prime}$ is any field extension of $K$, the group $\mathrm{G}_{\text {mot }}\left(\mathcal{M}_{K^{\prime}}\right)$ is a subgroup of finite index of $\mathrm{G}_{\mathrm{mot}}(\mathcal{M})$, and there exists a finite field extension $K^{\dagger}$ of $K$ such that for any extension $K^{\prime} / K^{\dagger}$ we have $\mathrm{G}_{\text {mot }}\left(\mathcal{M}_{K^{\prime}}\right)=\mathrm{G}_{\text {mot }}\left(\mathcal{M}_{K^{\dagger}}\right)$, see [22, Sect. 3.1]. In particular $\mathrm{G}_{\text {mot }}\left(\mathcal{M}_{\mathbb{C}}\right)$ is a subgroup of finite index of $\mathrm{G}_{\mathrm{mot}}(\mathcal{M})$. 


\subsection{Families of motives}

We will need to work with motives in families. For our purposes, it will be sufficient to treat the case in which the base field is $\mathbb{C}$. Let $S$ be a non-singular and connected complex quasiprojective variety. We will use the category $\mathrm{AM} / S$ of families of motives over $S$ defined by Moonen [22]. The category $\mathrm{AM} / S$ is a semisimple, neutral tannakian, abelian category, and there is a realization functor $r: \mathrm{AM} / S \rightarrow \mathrm{VHS}_{\mathbb{Q}} / S$ to polarized variations of $\mathbb{Q}$-Hodge structures over $S$.

The prototype of an object of AM/S is the motive $\mathcal{H}_{X / S}^{*}=\bigoplus_{j} \mathcal{H}_{X / S}^{j}$ of a smooth and projective morphism $\mathfrak{X} \rightarrow S$. For any $s \in S$ the fibre of $\mathcal{H}_{\mathfrak{X} / S}^{*}$ at $s$ is the motive $\mathcal{H}_{\mathfrak{X} s}^{*}$ of the fibre. The realization of the motive $\mathcal{H}_{X / S}^{*}$ is the variation of Hodge structures $H_{\mathfrak{X} / S}^{*}$ over $S$. In general, an object $\mathcal{M} / S \in \mathrm{AM} / S$ is cut out inside some $\mathcal{H}_{\mathfrak{X} / S}^{*}$ as above by a global section $p$ of the local system $\operatorname{End}(r(M) / S)$ such that $p \circ p=p$ and $p_{s}$ is a motivated cycle for all $s \in S$.

Let $\mathcal{M} / S \in \mathrm{AM} / S$ and let $M / S \in \mathrm{VHS}_{\mathbb{Q}} / S$ be its realization. We denote by $\mathcal{M}_{s}$ and $M_{S}$ the fibre at $s$ of $\mathcal{M} / S$ and $M / S$ respectively. Then there are local systems of algebraic groups over $S$

$$
G_{\text {mono }}(M / S)^{0} \unlhd \mathrm{MT}(M / S) \subset \mathrm{G}_{\mathrm{mot}}(\mathcal{M} / S) \subset \mathrm{GL}(M / S),
$$

where:

- $G_{\text {mono }}(M / S)^{0}$ is the connected monodromy group: for each $s \in S$ the fibre $G_{\text {mono }}(M / S)_{s}^{0}$ is the identity component of the Zariski closure of the image of the monodromy representation $\pi_{1}(S, s) \rightarrow \mathrm{GL}\left(M_{S}\right)$;

- $\operatorname{MT}(M / S)$ is the generic Mumford-Tate group: for each $s \in S$ we have an inclusion $\operatorname{MT}\left(M_{s}\right) \subset \operatorname{MT}(M / S)_{s}$, and equality holds for very general $s$, that is, for $s$ in the complement of a countable union of closed subvarieties of $S$;

- $\mathrm{G}_{\text {mot }}\left(\mathcal{M}_{s}\right)$ is the generic motivic Galois group: for each $s \in S$ we have an inclusion $\mathrm{G}_{\text {mot }}\left(\mathcal{M}_{s}\right) \subset \mathrm{G}_{\text {mot }}(\mathcal{M} / S)_{s}$, and equality holds for very general $s$.

The key result needed to develop the above theory is André's deformation principle for motivated cycles [2, Théorème 0.5$]$.

\subsection{Defect groups of hyper-Kähler varieties}

We now review some of the results on the André motives of hyper-Kähler varieties which we obtained in [10]. To any complex hyper-Kähler variety $X$ with $b_{2}(X)>3$ we attached an algebraic group

$$
P(X) \subset \mathrm{G}_{\mathrm{mot}}\left(\mathcal{H}_{X}^{*}\right),
$$

called the defect group of $X$, that is defined as follows.

If the odd cohomology of $X$ is trivial, then $P(X)$ is simply defined as the kernel of the projection $\mathrm{G}_{\text {mot }}\left(\mathcal{H}_{X}^{*}\right) \rightarrow \mathrm{G}_{\text {mot }}\left(\mathcal{H}_{X}^{2}\right)$ corresponding to $\mathcal{H}_{X}^{2} \subset \mathcal{H}_{X}^{*}$.

If instead $X$ has non-trivial cohomology in some odd degree, we need to consider the abelian variety $A$ obtained from $H_{X}^{2}$ via the Kuga-Satake construction [8] in order to control the odd part of the cohomology. In the case when $X$ has non-trivial odd cohomology we will assume the following:

Assumption 4.2 The motive $\mathcal{H}_{A}^{1}$ belongs to the tannakian category of motives generated by $\mathcal{H}_{X}^{*}$ 
We then define the defect group $P(X)$ as the kernel of the induced projection $\mathrm{G}_{\text {mot }}\left(\mathcal{H}_{X}^{*}\right) \rightarrow$ $\mathrm{G}_{\text {mot }}\left(\mathcal{H}_{A}^{1}\right)$. We expect Assumption 4.2 to be always satisfied; for the only known class of hyper-Kähler varieties with odd cohomology, that is, the deformation class of generalized Kummer varieties, we verified this in [10]. In general, contrarily to what claimed in loc. cit., the question remains open, see the Erratum [9] where we show that this assumption is not necessary to define the defect group; Assumption 4.2 may be translated into a question on the representation of the LLV-Lie algebra on the odd cohomology.

By construction, $P(X)$ acts on $H_{X}^{*}$ via graded algebra automorphisms and it acts trivially on $H_{X}^{2}$. We summarize below the main properties of the defect group $P(X)$, which are proven in [10, Lemma 6.8, Theorems 6.9 and 6.12].

Theorem 4.3 Let $X$ be a complex hyper-Kähler variety with $b_{2}(X)>3$. If $X$ has non-trivial cohomology in odd degree, assume that it satisfies Assumption 4.2. Then:

(a) the action of $P(X)$ on $H_{X}^{*}$ commutes with the LLV-Lie algebra $\mathfrak{g}(X)$;

(b) we have $\mathrm{G}_{\mathrm{mot}}\left(\mathcal{H}_{X}^{*}\right)=P(X) \times \mathrm{MT}\left(H_{X}^{*}\right)$;

(c) if $\mathfrak{X} \rightarrow S$ is a smooth and projective morphism to a non-singular connected variety $S$ with fibres hyper-Kähler varieties with $b_{2}>3$, then $s \mapsto P\left(\mathfrak{X}_{s}\right)$ defines a local system of algebraic groups $P(\mathfrak{X} / S) \subset \mathrm{GL}\left(H_{\mathfrak{X} / S}^{*}\right)$ over $S$.

If $\mathfrak{X} \rightarrow S$ is a family as in (c), the decomposition in (b) spreads over $S$ and gives a decomposition at the level of local systems of algebraic groups

$$
\mathrm{G}_{\text {mot }}\left(\mathcal{H}_{\mathfrak{X} / S}^{*}\right)=P(\mathfrak{X} / S) \times \operatorname{MT}\left(H_{\mathfrak{X} / S}^{*}\right) .
$$

For all $s \in S$, the inclusion of $\mathrm{G}_{\text {mot }}\left(\mathcal{H}_{\mathfrak{X}_{s}}^{*}\right)$ into $\mathrm{G}_{\text {mot }}(\mathfrak{X} / S)_{s}$ is the direct product of $\operatorname{MT}\left(H_{\mathfrak{X}_{s}}^{*}\right) \subset \operatorname{MT}\left(H_{\mathfrak{X} / S}^{*}\right)_{s}$ and $P\left(\mathfrak{X}_{s}\right)=P(\mathfrak{X} / S)_{s}$. Since the connected component of the identity of the monodromy group is a subgroup of the generic Mumford-Tate group of the family and the latter commutes with the defect group, the local system $P(\mathfrak{X} / S)$ becomes constant after a finite base change $S^{\prime} \rightarrow S$.

Remark 4.4 Conjecturally, the group $P(X)$ is trivial for any hyper-Kähler variety $X$ with $b_{2}>3$. In fact, the triviality of the defect group is equivalent to the conjecture which says that $\operatorname{MT}\left(H_{X}^{*}\right)=\mathrm{G}_{\mathrm{mot}}\left(\mathcal{H}_{X}^{*}\right)$ (i.e. Hodge classes are motivated), which would be a consequence of the Hodge conjecture.

\subsection{Proof of the main result}

With these preliminaries behind us we can proceed towards the proof of Theorem 1.1. To start with, we will need a stronger version of the deformation invariance of defect groups from Theorem 4.3.(c).

With $X$ as in Theorem 4.3, we have a canonical surjective homomorphism $\pi_{X}$ : $\mathcal{G}_{\text {mot }}\left(\mathrm{AM}_{\mathbb{C}}\right) \rightarrow \mathrm{G}_{\text {mot }}\left(\mathcal{H}_{X}^{*}\right)$. We also let

$$
\operatorname{pr}_{X}: \mathcal{G}_{\text {mot }}\left(\mathrm{AM}_{\mathbb{C}}\right) \rightarrow P(X)
$$

be the composition of $\pi_{X}$ with the projection coming from the isomorphism $\mathrm{G}_{\mathrm{mot}}\left(\mathcal{H}_{X}^{*}\right)=$ $P(X) \times \operatorname{MT}\left(H_{X}^{*}\right)$. Via Tannaka duality, the projection $\operatorname{pr}_{X}$ corresponds to the subcategory of $\left\langle\mathcal{H}_{X}^{*}\right\rangle$ of motives on which $\mathrm{MT}\left(H_{X}^{*}\right)$ acts trivially, i.e. the motives in $\left\langle\mathcal{H}_{X}^{*}\right\rangle$ with realization a direct sum of trivial Hodge structures $\mathbb{Q}(0)$. 
Remark 4.5 Composing $\pi_{X}$ with the other projection we obtain

$$
p_{X}: \mathcal{G}_{\operatorname{mot}}\left(\mathrm{AM}_{\mathbb{C}}\right) \rightarrow \operatorname{MT}\left(H_{X}^{*}\right) .
$$

By the definition of the defect group this homomorphism corresponds via Tannaka duality to the subcategory $\left\langle\mathcal{H}_{X}^{2}\right\rangle$ of $\mathrm{AM}_{\mathbb{C}}$ if the odd cohomology of $X$ is trivial, and to the subcategory $\left\langle\mathcal{H}_{A}^{1}\right\rangle$ otherwise, where $A$ is the Kuga-Satake abelian variety associated to $H_{X}^{2}$. Since $b_{2}(X)>$ 3 , the work of André [1] ensures that the motive $\mathcal{H}_{X}^{2}$ is abelian, i.e. it belongs to the tannakian subcategory $\mathrm{AM}_{\mathbb{C}}^{\mathrm{ab}}$ of $\mathrm{AM}_{\mathbb{C}}$ generated by the motives of abelian varieties. Hence, in any case the homomorphism $p_{X}$ factors through the quotient homomorphism $\mathcal{G}_{\text {mot }}\left(\mathrm{AM}_{\mathbb{C}}\right) \rightarrow$ $\mathcal{G}_{\text {mot }}\left(\mathrm{AM}_{\mathbb{C}}^{\mathrm{ab}}\right)$, where $\mathcal{G}_{\text {mot }}\left(\mathrm{AM}_{\mathbb{C}}^{\mathrm{ab}}\right)$ is the motivic Galois group of the tannakian category $\mathrm{AM}_{\mathbb{C}}^{\mathrm{ab}}$ of abelian motives.

Proposition 4.6 Let $\mathfrak{X} \rightarrow S$ be a smooth projective family of hyper-Kähler varieties with $b_{2}>3$; if the odd cohomology of the fibres is not trivial, assume that they satisfy Assumption 4.2. Assume that the monodromy group $G_{\text {mono }}\left(H_{\mathfrak{X} / S}^{*}\right)$ is connected. Let a, $b$ be points in $S$. Choose a continous path $\gamma$ from a to $b$ and let $\Xi: P\left(\mathfrak{X}_{a}\right) \rightarrow P\left(\mathfrak{X}_{b}\right)$ be the isomorphism obtained via parallel transport along $\gamma$ in the local system $P(\mathfrak{X} / S)$. Then $\Xi$ does not depend on the choice of $\gamma$ and the diagram

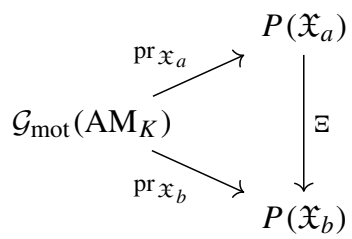

is commutative.

Proof Since the monodromy group is connected by assumption, the local system $P(\mathfrak{X} / S)$ is constant and $\Xi$ does not depend on the choice of the path $\gamma$.

Consider any motive over $S$ of the form

$$
\mathcal{T} / S:=\left(\mathcal{H}_{\mathfrak{X} / S}^{*}\right)^{\otimes t_{1}} \otimes\left(\mathcal{H}_{\mathfrak{X} / S}^{*}\right)^{\vee, \otimes t_{2}} \otimes \mathbb{Q}_{S}\left(t_{3}\right) \in \mathrm{AM} / S,
$$

for integers $t_{1}, t_{2}, t_{3}$. Let $T / S$ be its realization. For any $s \in S$ we let $W_{s} \subset T_{s}$ be the subspace of invariants for the generic Mumford-Tate group $\mathrm{MT}(T / S)_{S}$; this yields a sub-variation of Hodge structures $W / S \subset T / S$. Moreover, as $\operatorname{MT}(T / S)_{S}$ is normal in $\mathrm{G}_{\text {mot }}(\mathcal{T} / S)_{s}$, the variation $W / S$ is the realization of a submotive $\mathcal{W} / S \subset \mathcal{T} / S$ over $S$.

The motive $\mathcal{W} / S$ is a constant motive over $S$. Indeed, let us denote by $\mathcal{D}$ the motive $\mathcal{W}_{b}$, and let $\mathcal{D} / S$ be the constant motive over $S$ with fibre $\mathcal{D}$; let $D / S$ be the realization of $\mathcal{D} / S$. Then $\mathrm{id}_{b}: W_{b} \rightarrow D_{b}$ is monodromy invariant and obviously an isomorphism of motives; by [2, Théorème 0.5$]$ it extends to an isomorphism of families of motives $\mathcal{W} / S \stackrel{\sim}{\longrightarrow} \mathcal{D} / S$.

It follows that parallel transport along $\gamma$ in the local system $W / S$ gives a linear map $\Psi: W_{a} \rightarrow W_{b}$ which is the realization of an isomorphism of motives $\mathcal{W}_{a} \cong \mathcal{W}_{b}$. Hence the induced isomorphism $\Psi_{*}: \mathrm{GL}\left(W_{a}\right) \rightarrow \mathrm{GL}\left(W_{b}\right)$ fits into a commutative diagram

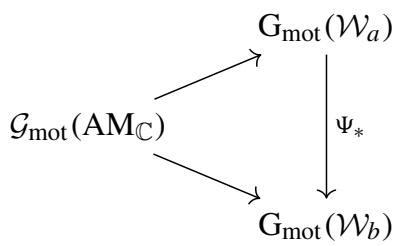


Note that since the generic Mumford-Tate group acts trivially on $W_{s}$ by construction, the group $\mathrm{G}_{\text {mot }}\left(\mathcal{W}_{s}\right)$ is a quotient of the defect group $P\left(\mathfrak{X}_{S}\right)$.

We now choose the tensor construction $T / S$ in such a way that the action of $P\left(\mathfrak{X}_{S}\right)$ on the subspace $W_{s}$ is faithful; in this case we have $\mathrm{G}_{\text {mot }}\left(\mathcal{W}_{s}\right)=P\left(\mathfrak{X}_{s}\right)$ for all points $s \in S$, and the homomorphism $\mathcal{G}_{\text {mot }}\left(\mathrm{AM}_{\mathbb{C}}\right) \rightarrow \mathrm{G}_{\text {mot }}\left(\mathcal{W}_{s}\right)$ is identified with $\mathrm{pr}_{\mathfrak{X}_{s}}: \mathcal{G}_{\text {mot }}\left(\mathrm{AM}_{\mathbb{C}}\right) \rightarrow P\left(\mathfrak{X}_{s}\right)$. Moreover, $P(\mathfrak{X} / S) \subset \mathrm{GL}(W / S)$ is a sub-local system of algebraic groups, and therefore the isomorphism $\Xi$ obtained via parallel transport along $\gamma$ in the local system $P(\mathfrak{X} / S)$ is the restriction of the isomorphism $\Psi_{*}: \operatorname{GL}\left(\mathcal{W}_{a}\right) \rightarrow \operatorname{GL}\left(\mathcal{W}_{b}\right)$ to $P\left(\mathfrak{X}_{a}\right)$. This concludes the proof.

By Remark 4.1, Theorem 1.1 is equivalent to the following statement.

Theorem 4.7 Let $X_{1}$ and $X_{2}$ be deformation equivalent complex hyper-Kähler varieties with $b_{2}>6$; if they have non-trivial odd cohomology, assume that they satisfy Assumption 4.2. Assume that $f: H_{X_{1}}^{2} \rightarrow H_{X_{2}}^{2}$ is a Hodge isometry. Then there exists an isomorphism of graded algebras $F: H_{X_{1}}^{*} \rightarrow H_{X_{2}}^{*}$ which is the realization of an isomorphism of motives $\mathcal{H}_{X_{1}}^{*} \rightarrow \mathcal{H}_{X_{2}}^{*}$.

Proof By Theorem 5.1 there exist smooth and projective families of hyper-Kähler varieties $\mathfrak{X}^{i} \rightarrow S_{i}$ over non-singular connected quasi-projective varieties $S_{i}$, for $i=1, \ldots, N$, and points $a_{i}, b_{i} \in S_{i}$ with isomorphisms

$$
X_{1} \stackrel{\sim}{\longrightarrow} \mathfrak{X}_{a_{1}}^{1}, \quad \mathfrak{X}_{b_{i}}^{i} \stackrel{\sim}{\longrightarrow} \mathfrak{X}_{a_{i+1}}^{i+1}, \text { for } i=1, \ldots, N-1, \quad \mathfrak{X}_{b_{N}}^{N} \stackrel{\sim}{\longrightarrow} X_{2} .
$$

We may and will assume that the monodromy groups $G_{\text {mono }}\left(H_{\mathfrak{X}^{i} / S_{i}}^{*}\right)$ are connected. For each $i$, let $\gamma_{i}$ be a path in $S_{i}$ from $a_{i}$ to $b_{i}$; let $\Psi: H_{X_{1}}^{*} \rightarrow H_{X_{2}}^{*}$ be the isomorphism obtained as composition of the isomorphisms $\Psi_{i}$ given by parallel transport along $\gamma_{i}$. We denote by $\psi:=\Psi^{(2)}: H_{X_{1}}^{2} \rightarrow H_{X_{2}}^{2}$ the isometry induced by $\Psi$.

Let $f: H_{X_{1}}^{2} \rightarrow H_{X_{2}}^{2}$ be a Hodge isometry. We construct the isomorphism of graded algebras $F: H_{X_{1}}^{*} \rightarrow H_{X_{2}}^{*}$ as in the proof of Proposition 3.4: we may assume that the isometry $\psi^{-1} \circ f: H_{X_{1}}^{2} \rightarrow H_{X_{1}}^{2}$ has determinant 1; thanks to Hilbert's Theorem 90 and the short exact sequence

$$
1 \rightarrow \mathbb{G}_{m} \rightarrow \operatorname{CSpin}\left(H_{X_{1}}^{2}\right) \stackrel{\pi}{\longrightarrow} \operatorname{SO}\left(H_{X_{1}}^{2}\right) \rightarrow 1,
$$

the morphism $\pi: \operatorname{CSpin}\left(H_{X_{1}}^{2}\right) \rightarrow \operatorname{SO}\left(H_{X_{1}}^{2}\right)$ is surjective on $\mathbb{Q}$-points and hence there exists $g \in \operatorname{CSpin}\left(H_{X_{1}}^{2}\right)(\mathbb{Q})$ such that $\pi(g)=\psi^{-1} \circ f$. By Lemma 2.6, $R(g)$ is a graded algebra automorphism of $H_{X_{1}}^{*}$, and we define

$$
F:=\Psi \circ R(g): H_{X_{1}}^{*} \rightarrow H_{X_{2}}^{*} .
$$

We claim that $F$ is the realization of an isomorphism of motives $\mathcal{H}_{X_{1}}^{*} \rightarrow \mathcal{H}_{X_{2}}^{*}$. If $F_{*}: \operatorname{GL}\left(H_{X_{1}}^{*}\right) \rightarrow \mathrm{GL}\left(H_{X_{2}}^{*}\right)$ denotes the induced isomorphism, we have to prove that $F_{*}$ restricts to an isomorphism $\mathrm{G}_{\mathrm{mot}}\left(\mathcal{H}_{X_{1}}^{*}\right) \stackrel{\sim}{\longrightarrow} \mathrm{G}_{\text {mot }}\left(\mathcal{H}_{X_{2}}^{*}\right)$ such that the diagram

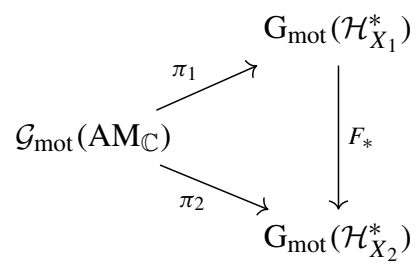


is commutative.

By Theorem 4.3.(a), the automorphism $R(g)$ of $H_{X_{1}}^{*}$ commutes with $P\left(X_{1}\right)$. Since $\Psi$ is the composition of parallel transport operators and the defect group is constant in families, $F_{*}$ identifies $P\left(X_{1}\right)$ with $P\left(X_{2}\right)$, and the induced isomorphism $F_{*}: P\left(X_{1}\right) \rightarrow P\left(X_{2}\right)$ is the restriction to $P\left(X_{1}\right)$ of $\Psi_{*}: \operatorname{GL}\left(H_{X_{1}}^{*}\right) \rightarrow \operatorname{GL}\left(H_{X_{2}}^{*}\right)$.

Since the degree 2 component of $F$ is a multiple of the Hodge isometry $f$, by Proposition 2.9, $F$ is an isomorphism of Hodge structures, and hence $F_{*}$ identifies $\operatorname{MT}\left(H_{X_{1}}^{*}\right)$ with $\operatorname{MT}\left(H_{X_{2}}^{*}\right)$. By Theorem 4.3.(b), $\mathrm{G}_{\mathrm{mot}}\left(\mathcal{H}_{X_{i}}^{*}\right)=P\left(X_{i}\right) \times \operatorname{MT}\left(H_{X_{i}}^{*}\right)$, and

it suffices to show the commutativity of the two diagrams
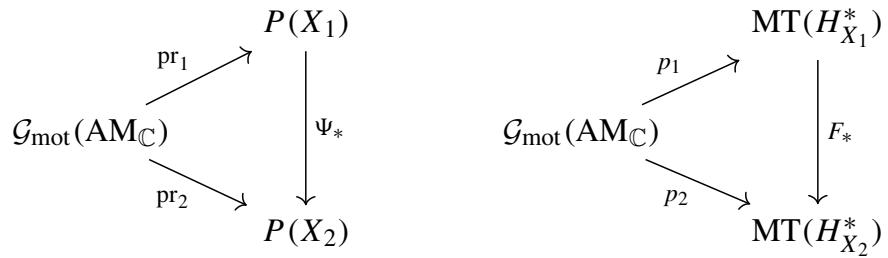

The left triangle is commutative by repeated application of Proposition 4.6, since the restriction of $\Psi_{*}$ to $P\left(X_{1}\right)$ is the composition of the isomorphisms $\Xi_{i}$ obtained via parallel transport along $\gamma_{i}$ in the local system $P\left(\mathfrak{X}^{i} / S_{i}\right)$.

For the right one, we proceed as follows. By Remark 4.5, $p_{i}$ corresponds to the inclusion of a subcategory of abelian motives; equivalently, for $i=1,2$, the homomorphism $p_{i}$ factors through $\mathcal{G}_{\text {mot }}\left(\mathrm{AM}_{\mathbb{C}}\right) \rightarrow \mathcal{G}_{\text {mot }}\left(\mathrm{AM}_{\mathbb{C}}^{\mathrm{ab}}\right)$. If we let $\mathrm{HS}_{\mathbb{Q}}^{\mathrm{ab}} \subset \mathrm{HS}_{\mathbb{Q}}^{\text {pol }}$ be the tannakian subcategory generated by the Hodge structures of abelian varieties, we have $\mathcal{M T}\left(\mathrm{HS}_{\mathbb{Q}}^{\mathrm{ab}}\right)=\mathcal{G}_{\operatorname{mot}}\left(\mathrm{AM}_{\mathbb{C}}^{\mathrm{ab}}\right)$, by [2, Théorème 0.6.4]. But now the diagram

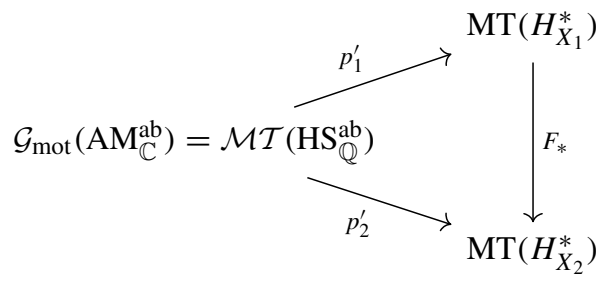

is commutative, since $F$ is an isomorphism of Hodge structures.

Finally, we prove Corollary 1.2 from the introduction.

Corollary 4.8 Let $K \subset \mathbb{C}$ be a subfield which is finitely generated over $\mathbb{Q}$, and let $X_{1}, X_{2}$ be deformation equivalent hyper-Kähler varieties over $K$ with $b_{2}\left(X_{i}\right)>6$. If their odd cohomology is not trivial, assume that Assumption 4.2 holds for $X_{1}$ and $X_{2}$. Assume that $f: H_{X_{1}, \ell}^{2} \rightarrow H_{X_{2}, \ell}^{2}$ is a $\operatorname{Gal}(\bar{K} / K)$-equivariant isometry. Then, there exist a finite field extension $K^{\prime} / K$ and an isomorphism $F: H_{X_{1}, \ell}^{*} \rightarrow H_{X_{2}, \ell}^{*}$ of graded algebras which is $\operatorname{Gal}\left(\bar{K} / K^{\prime}\right)$-equivariant.

Proof By [1], the Mumford-Tate conjecture in degree 2 holds for $X_{1}$ and $X_{2}$, and the motives $\mathcal{H}_{X_{1}}^{2}$ and $\mathcal{H}_{X_{2}}^{2}$ are abelian. Hence, there exists a finite extension $K^{\prime}$ of $K$ such that the isometry $f$ is the realization of an isomorphism of motives over $K^{\prime}$ with $\mathbb{Q}_{\ell}$-coefficients. The same argument as in the proof of Theorem 4.7 produces an isomorphism of graded algebras $F: H_{X_{1}, \ell}^{*} \rightarrow H_{X_{2}, \ell}^{*}$ which, up to further replacing $K^{\prime}$ with a finite extension, is 
the realization of an isomorphism of motives over $K^{\prime}$ with $\mathbb{Q}_{\ell}$-coefficients. Hence, $F$ is $\operatorname{Gal}\left(\bar{K} / K^{\prime}\right)$-equivariant.

\section{Projective families of hyper-Kähler varieties}

In the proof of Theorem 1.1 we used the following result.

Theorem 5.1 Let $X_{1}, X_{2}$ be deformation equivalent complex projective hyper-Kähler varieties. Assume that $b_{2}\left(X_{i}\right)>6$. Then there exist:

- smooth and projective families $\mathfrak{X}^{i} \rightarrow S_{i}$ with fibres hyper-Kähler varieties over connected and non-singular quasi-projective complex varieties $S_{i}$, for $i=1, \ldots, N$;

- points $a_{i}, b_{i} \in S_{i}$, for $i=1, \ldots, N$, together with isomorphisms

$$
X_{1} \stackrel{\sim}{\longrightarrow} \mathfrak{X}_{a_{1}}^{1}, \quad \mathfrak{X}_{b_{i}}^{i} \stackrel{\sim}{\longrightarrow} \mathfrak{X}_{a_{i+1}}^{i+1}, \text { for } i=1, \ldots, N-1, \quad \mathfrak{X}_{b_{N}}^{N} \stackrel{\sim}{\longrightarrow} X_{2} .
$$

If $X_{1}$ and $X_{2}$ satisfy the conclusion of the Theorem, we will write $X_{1} \sim X_{2}$.

Remark 5.2 In similar spirit, Soldatenkov [28] shows that, under the assumption that $b_{2}>3$, the varieties $X_{1}$ and $X_{2}$ can be joined via smooth and proper (but not necessarily projective) families over curves; however, the total space of such a family is not an algebraic variety in general.

Before giving the proof of Theorem 5.1 we recall some facts on polarized hyper-Kähler varieties. The positive cone of a hyper-Kähler manifold $X$ is the connected component of $\{x \in$ $H^{1,1}(X, \mathbb{R})$ such that $\left.(x, x)>0\right\}$ containing the Kähler cone, where $(\cdot, \cdot)$ is the BeauvilleBogomolov pairing. We denote by $\mathrm{NS}^{+}(X) \subset \mathrm{NS}(X)$ the intersection of the positive cone with the Néron-Severi group. In analogy to the case of K3 surfaces and -2-classes, the ample cone $\operatorname{Amp}(X) \subset \mathrm{NS}^{+}(X)$ of a projective hyper-Kähler manifold can be described in terms of so-called MBM classes [3] (this notion is equivalent to that of wall divisors introduced in [21]). The proof of Theorem 5.1 relies on the following result due to Amerik and Verbitsky. Let $\operatorname{MBM}(X) \subset \mathrm{NS}(X)$ be the subset of MBM classes on $X$.

Theorem 5.3 (i) [3] Let $X$ be a projective hyper-Kähler manifold. The ample cone of $X$ is one of the connected components of

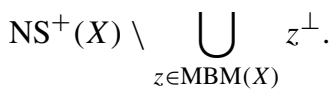

In particular, if $\operatorname{MBM}(X)=\emptyset$ then $\operatorname{Amp}(X)=\mathrm{NS}^{+}(X)$.

(ii) [4] Fix a deformation class of hyper-Kähler manifolds with $b_{2} \geq 5$. There exists a positive integer $N$, depending only on the deformation class, such that for any projective $X$ of the given deformation type, every MBM class $z$ on $X$ satisfies

$$
-N<(z, z)<0 .
$$

Let $X$ be a hyper-Kähler manifold and let $\Lambda$ be a lattice isometric to $H^{2}(X, \mathbb{Z})$ equipped with the Beauville-Bogomolov form. Let

$$
\Omega=\{x \in \mathbb{P}(\Lambda \otimes \mathbb{C}) \mid(x, x)=0,(x, \bar{x})>0\}
$$

be the period domain. We fix a connected component $\mathfrak{M}$ of the moduli space of $\Lambda$-marked hyper-Kähler manifolds containing $X$ (with a chosen marking). By the global Torelli theorem 
[17,31], the period map $\mathcal{P}: \mathfrak{M} \rightarrow \Omega$ is surjective with finite fibres, and each fibre consists of bimeromorphic hyper-Kähler manifolds.

By Huybrechts' projectivity criterion [15], a hyper-Kähler manifold $Y$ is projective if and only if $\mathrm{NS}(Y)$ contains a class $h$ with $(h, h)>0$. For any positive class $h \in \Lambda$, we have a hypersurface

$$
\Omega_{h^{\perp}}=\{x \in \Omega \mid(x, h)=0\} \subset \Omega .
$$

The period space $\Omega_{h^{\perp}}$ has two connected components; we denote by $\Omega_{h^{\perp}}^{+}$the component parametrizing those $(Y, \tau) \in \mathfrak{M}$ such that $\tau^{-1}(h)$ belongs to the positive cone.

We let $\mathfrak{M}_{h^{\perp}}^{\mathrm{a}} \subset \mathfrak{M}$ be the subset consisting of $(Y, \tau)$ such that $\tau^{-1}(h)$ represents an ample class on $Y$. Then $\mathfrak{M}_{h^{\perp}}^{\mathrm{a}}$ is Hausdorff and connected, and its image $\Omega_{h^{\perp}}^{\mathrm{a}}$ via the period map is open and dense in $\Omega_{h^{\perp}}^{+}$, by [20, Corollary 7.3].

\subsection{Proof of Theorem 5.1}

The rest of the article is devoted to the proof of Theorem 5.1. Let $X$ be a hyper-Kähler manifold and assume that $b_{2}(X)>6$. We fix a connected component $\mathfrak{M}$ of the moduli space of $\Lambda$-marked hyper-Kähler manifolds.

We will show that given any $\left(X_{1}, \tau_{1}\right)$ and $\left(X_{2}, \tau_{2}\right)$ in $\mathfrak{M}$ with $X_{1}$ and $X_{2}$ projective, then $X_{1} \sim X_{2}$, in the notation of the Theorem. We start with the following case.

Proposition 5.4 Let $h \in \Lambda$ be a positive class, and let $\left(X_{1}, \tau_{1}\right),\left(X_{2}, \tau_{2}\right)$ be points of $\mathfrak{M}_{h^{\perp}}^{\mathrm{a}}$. Then $X_{1} \sim X_{2}$.

Proof Let $(Y, L)$ be a hyper-Kähler variety equipped with an ample divisor $L$. Following André [1, Sect. 3.3] (see also [14]) there exists a polarized deformation $\mathfrak{Y} \rightarrow S$ of $(Y, L)$ which is a smooth and projective family of hyper-Kähler varieties over a non-singular and connected quasi-projective variety $S$, with a distinguished fibre $\mathfrak{Y}_{s}=Y$ and the following property: denoting by $\tilde{S} \rightarrow S$ the universal covering of $S$, the image of the period map $\tilde{S} \rightarrow \Omega_{h^{\perp}}^{\mathrm{a}}$ contains an open subset. Here, $h=\phi\left(c_{1}(L)\right)$ for a fixed marking $\phi$ on $Y$. Upon replacing $S$ with a finite cover, we find a torsion free arithmetic subgroup $\Gamma \subset \mathrm{O}\left(h^{\perp}\right)$ acting freely on $\Omega_{h^{\perp}}^{+}$such that the period map descends to $\Psi: S \rightarrow \Gamma \backslash \Omega_{h^{\perp}}^{+}$. By [6], $\Gamma \backslash \Omega_{h^{\perp}}^{+}$is a non-singular quasi-projective variety, and the map $\Psi$ is a dominant morphism of algebraic varieties.

Let now $\left(X_{1}, \tau_{1}\right)$ and $\left(X_{2}, \tau_{2}\right)$ be as in the statement of the proposition. Consider the respective polarized deformations $\mathfrak{X}_{1} \rightarrow S_{1}$ and $\mathfrak{X}_{2} \rightarrow S_{2}$ of $\left(X_{1}, \tau_{1}^{-1}(h)\right)$ and $\left(X_{2}, \tau_{2}^{-1}(h)\right)$ described above. For a suitable torsion free arithmetic subgroup $\Gamma \subset \mathrm{O}\left(h^{\perp}\right)$ and finite covers of $S_{1}$ and $S_{2}$, we have dominant period maps $\Psi_{1}: S_{1} \rightarrow \Gamma \backslash \Omega_{h^{\perp}}^{+}$and $\Psi_{2}: S_{2} \rightarrow \Gamma \backslash \Omega_{h^{\perp}}^{+}$. As $\Omega_{h^{\perp}}^{+}$is connected, $\Psi_{1}\left(S_{1}\right) \cap \Psi_{2}\left(S_{2}\right)$ is not empty. By the surjectivity of the period map, there exists $(Y, \tau) \in \mathfrak{M}$ whose period gives a point in this intersection via the quotient map $\Omega_{h^{\perp}}^{+} \rightarrow \Gamma \backslash \Omega_{h^{\perp}}^{+}$; if $(Y, \tau)$ is very general with the above property, then $Y$ is of Picard rank 1. In this case $(Y, \tau)$ is the unique point in the fibre of the period map containing it, and it belongs to $\mathfrak{M}_{h^{\perp}}^{\mathrm{a}}$. It follows that there exist $s_{1} \in S_{1}$ and $s_{2} \in S_{2}$ and an isomorphism between the fibres $\mathfrak{X}_{1, s_{1}} \cong Y \cong \mathfrak{X}_{2, s_{2}}$.

The next case is the key step in the proof. 
Proposition 5.5 Let $h_{1}$ and $h_{2}$ be positive classes in $\Lambda$ such that the lattice $\left\langle h_{1}, h_{2}\right\rangle$ is of signature $(1,1)$ and $\left(h_{1}, h_{2}\right)>0$. Assume that $\left(X_{1}, \tau_{1}\right) \in \mathfrak{M}_{h_{1}^{\perp}}^{\mathrm{a}}$ and $\left(X_{2}, \tau_{2}\right) \in \mathfrak{M}_{h_{2}^{\perp}}^{\mathrm{a}}$. Then $X_{1} \sim X_{2}$.

Proof Let $h_{1}$ and $h_{2}$ be as above. Then $\Omega_{h_{1}^{\perp}}^{+} \cap \Omega_{h_{2}^{\perp}}^{+} \neq \emptyset$. If $\mathfrak{M}_{h_{1}^{\perp}}^{\mathrm{a}} \cap \mathfrak{M}_{h_{2}^{\perp}}^{\mathrm{a}}$ is also not empty, we can directly apply Proposition 5.4 to conclude; however in general this is not the case, and we need to modify $h_{1}$ and $h_{2}$ before applying that proposition.

We fix the constant $N$ given by Theorem 5.3 for our deformation type. First of all, we replace $h_{1}$ and $h_{2}$ with classes of which we can control the square. There exist $\left(Y_{1}, \phi_{1}\right) \in \mathfrak{M}_{h_{1}^{\perp}}^{\mathrm{a}}$ and $\left(Y_{2}, \phi_{2}\right) \in \mathfrak{M}_{h_{2}^{\perp}}^{\mathrm{a}}$ of maximal Picard rank. Then $\mathrm{NS}\left(Y_{i}\right)$ is an indefinite lattice of rank $b_{2}-2 \geq 5$, and hence it contains a non-zero isotropic vector $\phi_{i}^{-1}\left(y_{i}\right) \in \mathrm{NS}\left(Y_{i}\right)$, for $i=1,2$.

Lemma 5.6 There exist a prime number $p>N$ congruent to 3 modulo 4 , an odd integer $j \gg 0$ and positive classes $l_{1}$ and $l_{2}$ in $\Lambda$ such that:

- $\phi_{1}^{-1}\left(l_{1}\right)$ (resp. $\left.\phi_{2}^{-1}\left(l_{2}\right)\right)$ represents an ample divisor on $Y_{1}$ (resp. $\left.Y_{2}\right)$;

- $\left(l_{1}, l_{1}\right)=p^{j} f_{1}$ and $\left(l_{2}, l_{2}\right)=p^{j} f_{2}$, with $f_{1}$ and $f_{2}$ not divisible by $p$ and such that $f_{1}$ and $f_{2}$ are both quadratic residues modulo $p$;

- the lattice generated by $l_{1}$ and $l_{2}$ has signature $(1,1)$, and $\left(l_{1}, l_{2}\right)>0$.

Proof Choose a large prime $p>N$ congruent to 3 modulo 4 and which does not divide any of the integers $\left(h_{1}, h_{1}\right),\left(h_{2}, h_{2}\right),\left(h_{1}, y_{1}\right)$ and $\left(h_{2}, y_{2}\right)$. Replacing $y_{i}$ with a suitable multiple, we may assume that $2\left(h_{1}, y_{1}\right)$ and $2\left(h_{2}, y_{2}\right)$ are (non-zero) quadratic residues modulo $p$. Consider

$$
l_{1}:=p^{j} h_{1}+y_{2} \text { and } l_{2}:=p^{j} h_{2}+y_{2} .
$$

Then we have

$$
\begin{aligned}
& \left(l_{1}, l_{1}\right)=p^{j}\left(p^{j}\left(h_{1}, h_{1}\right)+2\left(h_{1}, y_{1}\right)\right)=: p^{j} f_{1}, \\
& \left(l_{2}, l_{2}\right)=p^{j}\left(p^{j}\left(h_{2}, h_{2}\right)+2\left(h_{2}, y_{2}\right)\right)=: p^{j} f_{2} .
\end{aligned}
$$

For a large enough odd integer $j$, the class $\phi_{1}^{-1}\left(l_{1}\right)$ (resp. $\left.\phi_{2}^{-1}\left(l_{2}\right)\right)$ represents an ample divisor on $Y_{1}$ (resp. $\left.Y_{2}\right)$. Moreover $\left\langle l_{1}, l_{2}\right\rangle$ has signature $(1,1)$ and $\left(l_{1}, l_{2}\right)>0$. Therefore $l_{1}$ and $l_{2}$ have the required properties.

Thanks to Proposition 5.4 it suffices to prove Proposition 5.5 for $l_{i}, Y_{i}, \phi_{i}$ as above in place of $h_{i}, X_{i}, \tau_{i}$.

Lemma 5.7 There exist vectors $v_{1} \in l_{1}^{\perp}$ and $v_{2} \in l_{2}^{\perp}$ such that:

- $\left(v_{1}, v_{1}\right)=p^{r_{1}} \epsilon_{1}$, where $r_{1}$ is an odd natural number and $\epsilon_{1}$ is a negative integer not divisible by $p$ and it is a quadratic residue modulo $p$;

- $\left(v_{2}, v_{2}\right)=p^{r_{2}} \epsilon_{2}$, where $r_{2}$ is an odd natural number and $\epsilon_{2}$ is a negative integer not divisible by $p$ and it is a quadratic residue modulo $p$;

$-\left(v_{1}, v_{2}\right)=0=\left(l_{2}, v_{1}\right)$.

Proof The orthogonal to $\left\langle l_{1}, l_{2}\right\rangle$ in $\Lambda$ is an indefinite lattice of rank at least 5; hence, we can find isotropic vectors $u_{1}, u_{2} \in\left\langle l_{1}, l_{2}\right\rangle^{\perp}$ such that $\left(u_{1}, u_{2}\right)=t<0$. The self-intersection of a linear combination $a u_{1}+b u_{2}$ is $2 a b t$. It is then clear that a suitable linear combination $v_{1}$ 
of $u_{1}$ and $u_{2}$ will satisfy $\left(v_{1}, v_{1}\right)=p^{r_{1}} \epsilon_{1}$ for some odd natural number $r_{1}$ and a negative integer $\epsilon_{1}$ which is a non-zero square modulo $p$.

The orthogonal to $\left\langle l_{2}, v_{1}\right\rangle$ is an indefinite lattice of rank at least 5; hence, it contains isotropic vectors $z_{1}, z_{2} \in\left\langle l_{2}, v_{1}\right\rangle^{\perp}$ such that $\left(z_{1}, z_{2}\right)=s<0$. As above, a suitable linear combination $v_{2}$ of $z_{1}$ and $z_{2}$ satisfies $\left(v_{2}, v_{2}\right)=p^{r_{2}} \epsilon_{2}$, for some odd natural number $r_{2}$ and a negative integer $\epsilon_{2}$ which is a non-zero square modulo $p$.

Consider now the rank 2 sub-lattices of $\Lambda$ :

$$
L_{1}=\left\langle l_{1}, v_{1}\right\rangle \text { and } L_{2}=\left\langle l_{2}, v_{2}\right\rangle .
$$

The proof of the next Claims 5.8 and 5.9 below will be given at the end of the section.

Claim 5.8 For $i=1$ or 2 , let $v \in L_{i} \otimes \mathbb{Q}$. If $(v, v) \in \mathbb{Z}$, then $p$ divides $(v, v)$.

Since the lattices $L_{1}$ and $L_{2}$ are of signature $(1,1)$, there exist $\left(W_{1}, \psi_{1}\right)$ and $\left(W_{2}, \psi_{2}\right)$ in $\mathfrak{M}$ such that $\mathrm{NS}\left(W_{1}\right)=\psi_{1}^{-1}\left(L_{1}\right)$ and $\operatorname{NS}\left(W_{2}\right)=\psi_{2}^{-1}\left(L_{2}\right)$ and $\psi_{1}^{-1}\left(l_{1}\right)$ (resp. $\psi_{2}^{-1}\left(l_{2}\right)$ ) belongs to $\mathrm{NS}^{+}\left(W_{1}\right)$ (resp. $\left.\mathrm{NS}^{+}\left(W_{2}\right)\right)$. Claim 5.8 ensures that $\mathrm{NS}\left(W_{1}\right)$ and $\mathrm{NS}\left(W_{2}\right)$ contain no MBM classes; hence, by Theorem 5.3,

$$
\operatorname{Amp}\left(W_{1}\right)=\mathrm{NS}^{+}\left(W_{1}\right) \text { and } \operatorname{Amp}\left(W_{2}\right)=\mathrm{NS}^{+}\left(W_{2}\right) .
$$

In particular, $\psi_{1}^{-1}\left(l_{1}\right)$ (resp. $\left.\psi_{2}^{-1}\left(l_{2}\right)\right)$ represents an ample class on $W_{1}\left(\right.$ resp. $\left.W_{2}\right)$.

For $k>0$, we define $w_{1, k} \in L_{1}$ and $w_{2, k} \in L_{2}$ as:

$$
w_{1, k}:=p^{k} l_{1}+v_{1} \quad w_{2, k}:=p^{k} l_{2}+v_{2} .
$$

For $k$ large enough, $\psi_{1}^{-1}\left(w_{1, k}\right)$ (resp. $\left.\psi_{2}^{-1}\left(w_{2, k}\right)\right)$ represents an ample class on $W_{1}$ (resp. on $W_{2}$ ). We let $S_{k} \subset \Lambda$ be the lattice generated by $w_{1, k}$ and $w_{2, k}$.

Claim 5.9 Let $v \in S_{k} \otimes \mathbb{Q}$, for $k \gg 0$. If $(v, v) \in \mathbb{Z}$, then $p$ divides $(v, v)$.

If $k \gg 0$, the lattice $S_{k}$ has signature $(1,1)$, because this is the signature of the lattice generated by $l_{1}$ and $l_{2}$. By the surjectivity of the period map, there exists $(Z, \eta) \in \mathfrak{M}$ such that $\operatorname{NS}(Z)=\eta^{-1}\left(S_{k}\right)$ and both the classes $\eta^{-1}\left(w_{1, k}\right)$ and $\eta^{-1}\left(w_{2, k}\right)$ belong to $\mathrm{NS}^{+}(Z)$; this is possible because $\left(w_{1, k}, w_{2, k}\right)>0$ for $k \gg 0$.

By Claim 5.9, there are no MBM classes in $\mathrm{NS}(Z)$; hence, $\operatorname{Amp}(Z)=\mathrm{NS}^{+}(Z)$, by Theorem 5.3. We therefore obtain:

$$
\left(W_{1}, \psi_{1}\right) \in \mathfrak{M}_{l_{1}^{\perp}}^{\mathrm{a}} \cap \mathfrak{M}_{w_{1, k}^{\perp}}^{\mathrm{a}}, \quad(Z, \eta) \in \mathfrak{M}_{w_{1, k}^{\perp}}^{\mathrm{a}} \cap \mathfrak{M}_{w_{2, k}^{\perp}}^{\mathrm{a}}, \quad\left(W_{2}, \psi_{2}\right) \in \mathfrak{M}_{w_{2, k}^{\perp}}^{\mathrm{a}} \cap \mathfrak{M}_{l_{2}^{\perp}}^{\mathrm{a}} .
$$

By Proposition 5.4, this concludes the proof of Proposition 5.5.

Finally, Proposition 5.5 implies Theorem 5.1 via the next lemma.

Lemma 5.10 Let $h_{1}, h_{2}$ be linearly independent positive classes in $\Lambda$. Then there exist finitely many vectors $l_{1}, l_{2}, \ldots, l_{k} \in \Lambda$ such that:

$-l_{1}=h_{1}$ and $l_{k}=h_{2}$;

- $\left(l_{i}, l_{i}\right)>0$, for each $i=1, \ldots, k$;

- $\left\langle l_{i}, l_{i+1}\right\rangle$ has signature $(1,1)$ and $\left(l_{i}, l_{i+1}\right)>0$, for each $i=1, \ldots, k-1$.

Proof The argument presented here is due to Soldatenkov [28, Sect. 6.3]. If $\left\langle h_{1}, h_{2}\right\rangle$ is of signature $(1,1)$ and $\left(h_{1}, h_{2}\right)>0$ there is nothing to do.

Assume that $\left\langle h_{1}, h_{2}\right\rangle$ is positive definite. We may assume that $\left(h_{1}, h_{2}\right)=0$, for, otherwise, we pick a positive class $h_{3} \in\left\langle h_{1}, h_{2}\right\rangle^{\perp}$ and apply the argument below to $h_{1}, h_{3}$ and $h_{3}, h_{2}$. Consider $V$ consisting of $l \in \Lambda \otimes \mathbb{R}$ such that: 
$-(l, l)>0,\left(h_{1}, l\right)>0$ and $\left(h_{2}, l\right)>0$;

- $\left\langle h_{1}, l\right\rangle$ and $\left\langle l, h_{2}\right\rangle$ are both of signature $(1,1)$.

Then $V$ is an open cone in $\Lambda \otimes \mathbb{R}$ and it suffices to show that $V$ is not empty. Let $w \in\left\langle h_{1}, h_{2}\right\rangle^{\perp}$ be such that $(w, w)<0$ and let $u_{1}, u_{2}, u_{3}$ be the orthogonal basis of $\left\langle h_{1}, h_{2}, w\right\rangle \otimes \mathbb{R}$ such that $\left(u_{1}, u_{1}\right)=1,\left(u_{2}, u_{2}\right)=1,\left(u_{3}, u_{3}\right)=-1$, and $h_{1}=\alpha u_{1}, h_{2}=\beta u_{2}, w=\gamma u_{3}$, for positive real numbers $\alpha, \beta$ and $\gamma$. A computation shows that the vector $l=u_{1}+u_{2}+\delta u_{3}$ is positive for $\delta^{2}<2$, and both the real vector spaces $\left\langle h_{1}, l\right\rangle$ and $\left\langle h_{2}, l\right\rangle$ are of signature $(1,1)$ for $\delta^{2}>1$. Moreover, $\left(h_{1}, l\right)=\alpha$ and $\left(h_{2}, l\right)=\beta$ are positive. Hence, if $1<\delta^{2}<2$, the vector $l \in V$.

If $\left\langle h_{1}, h_{2}\right\rangle$ is of signature $(1,1)$ and $\left(h_{1}, h_{2}\right)<0$, we simply let $l$ be a positive class in $\left\langle h_{1}, h_{2}\right\rangle^{\perp}$. Then $\left\langle h_{1}, l\right\rangle$ and $\left\langle h_{2}, l\right\rangle$ are positive definite, and we conclude as above.

Finally, let $\left\langle h_{1}, h_{2}\right\rangle$ be degenerate. The set $V$ of $l \in \Lambda \otimes \mathbb{R}$ such that

- $(l, l)>0$, and

- $\left\langle h_{1}, l\right\rangle$ and $\left\langle l, h_{2}\right\rangle$ are both non-degenerate,

is a non-empty open cone in $\Lambda \otimes \mathbb{R}$. Hence, there exists a positive class $l \in \Lambda$ such that $\left\langle h_{1}, l\right\rangle$ and $\left\langle l, h_{2}\right\rangle$ are both non-degenerate. This concludes the proof.

Below are proven the statements used in the proof of Proposition 5.5.

Proof (of Claim 5.8) By construction, the intersection matrices of $L_{1}$ and $L_{2}$ are

$$
\left(\begin{array}{cc}
p^{j} f_{1} & 0 \\
0 & p^{r_{1}} \epsilon_{1}
\end{array}\right) \text { and }\left(\begin{array}{cc}
p^{j} f_{2} & 0 \\
0 & p^{r_{2}} \epsilon_{2}
\end{array}\right),
$$

respectively, with $j \gg 0$ odd and $f_{1}, f_{2}, \epsilon_{1}, \epsilon_{2}$ all non-zero squares modulo $p$. In particular, $\epsilon_{1} f_{1}$ and $\epsilon_{2} f_{2}$ are squares modulo $p$.

Let $v \in L_{1} \otimes \mathbb{Q}$; the case of $v \in L_{2} \otimes \mathbb{Q}$ is analogous. Assume that $(v, v)$ is an integer. There exist integers $\gamma, \lambda, \delta$ such that $\gamma v=\lambda l_{1}+\delta v_{1}$. Assume that $j \geq r_{1}$ (otherwise we proceed similarly). We then have

$$
\gamma^{2}(v, v)=p^{r_{1}}\left(\lambda^{2} p^{j-r_{1}} f_{1}+\delta^{2} \epsilon_{1}\right) .
$$

Assume by contradiction that $(v, v)$ is not divisible by $p$, and let $m$ be the biggest integer such that $p^{m}$ divides both $\lambda$ and $\delta$. We can then write

$$
\gamma^{2}(v, v)=p^{2 m+r_{1}}\left(\lambda_{0}^{2} p^{j-r_{1}} f_{1}+\delta_{0}^{2} \epsilon_{1}\right),
$$

where $p$ does not divide both $\lambda_{0}$ and $\delta_{0}$. The left hand-side is divisible by an even power of $p$. Since $r_{1}$ is odd, if $j-r_{1}>0$ this forces $\delta_{0}$ to be divisible by $p$, and hence $\delta_{0}=p \delta_{1}$ for some integer $\delta_{1}$. Therefore, $\lambda_{0}$ is not divisible by $p$. We obtain

$$
\gamma^{2}(v, v)=p^{2 m+r_{1}+2}\left(\lambda_{0}^{2} p^{j-r_{1}-2} f_{1}+\delta_{1}^{2} \epsilon_{1}\right) .
$$

If $j-r_{1}-2>0$, we again find that $p$ has to divide $\delta_{1}$, so $\delta_{0}=p^{2} \delta_{2}$ and

$$
\gamma^{2}(v, v)=p^{2 m+r_{1}+4}\left(\lambda_{0}^{2} p^{j-r_{1}-4} f_{1}+\delta_{2}^{2} \epsilon_{1}\right) .
$$

Proceeding in this way we eventually find $\bar{\delta}$ such that $\delta_{0}=p^{\left(j-r_{1}\right) / 2} \bar{\delta}$ and

$$
\gamma^{2}(v, v)=p^{2 m+j}\left(\lambda_{0}^{2} f_{1}+\bar{\delta}^{2} \epsilon_{1}\right) .
$$

Now, since $j$ is odd, $p$ has to divide $\lambda_{0}^{2} f_{1}+\bar{\delta}^{2} \epsilon_{1}$. But $-f_{1} \epsilon_{1}$ is not a square modulo $p$ because $p \equiv 3$ modulo 4 , and therefore $\lambda_{0}^{2} f_{1}+\bar{\delta}^{2} \epsilon_{1} \equiv 0$ modulo $p$ has no non-trivial solutions. It follows that $p$ divides $\lambda_{0}$, a contradiction. 
Proof (of Claim 5.9) We let $b:=\left(l_{1}, l_{2}\right)$ and $s:=\left(l_{1}, v_{2}\right)$. The intersection matrix of $S_{k}$ is

$$
\left(\begin{array}{cc}
p^{2 k+j} f_{1}+p^{r_{1}} \epsilon_{1} & p^{2 k} b+p^{k} s \\
p^{2 k} b+p^{k} s & p^{2 k+j} f_{2}+p^{r_{2}} \epsilon_{2}
\end{array}\right) .
$$

Let $v \in S_{k} \otimes \mathbb{Q}$ be such that $(v, v) \in \mathbb{Z}$. We have $\gamma v=\lambda w_{1, k}+\delta w_{2, k}$ for integers $\gamma, \lambda, \delta$. Without loss of generality, we may assume that $r_{1} \leq r_{2}$. Then:

$$
\gamma^{2}(v, v)=p^{r_{1}}\left(\lambda^{2} \epsilon_{1}+\delta^{2} p^{r_{2}-r_{1}} \epsilon_{2}+D\right)
$$

where

$$
D=\lambda^{2} p^{2 k+j-r_{1}} f_{1}+2 \lambda \delta\left(p^{2 k-r_{1}} b+p^{k-r_{1}} s\right)+\delta^{2} p^{2 k+j-r_{1}} f_{2}
$$

is divisible by a large power of $p$, since $k \gg 0$. Let $m$ be such that $\lambda=p^{m} \lambda_{0}$ and $\delta=p^{m} \delta_{0}$, with at least one among $\lambda_{0}$ and $\delta_{0}$ not divisible by $p$. We can then write

$$
\gamma^{2}(v, v)=p^{2 m+r_{1}}\left(\lambda_{0}^{2} \epsilon_{1}+\delta_{0}^{2} p^{r_{2}-r_{1}} \epsilon_{2}+D_{0}\right) .
$$

Assume by contradiction that $p$ does not divide $(v, v)$. Then $\gamma^{2}(v, v)$ is divisible by an even power of $p$, and hence, being $r_{1}$ odd, $p$ necessarily divides the term in brackets on the right hand side. If $r_{2}>r_{1}$ this forces $\lambda_{0}$ to be divisible by $p$, so $\lambda_{0}=p \lambda_{1}$. Proceeding as in the previous proof, we obtain $\bar{\lambda}$ such that $\lambda_{0}=p^{\left(r_{2}-r_{1}\right) / 2} \bar{\lambda}$ and

$$
\gamma^{2}(v, v)=p^{2 m+r_{2}}\left(\bar{\lambda}^{2} \epsilon_{1}+\delta_{0}^{2} \epsilon_{2}+D_{0} / p^{r_{2}-r_{1}}\right),
$$

Note that $D_{0} / p^{r_{2}-r_{1}}$ is still an integer divisible by $p$. But then we must have

$$
\bar{\lambda}^{2} \epsilon_{1}+\delta_{0}^{2} \epsilon_{2} \equiv 0 \text { modulo } p \text {. }
$$

Since $\epsilon_{1} \epsilon_{2}$ is a square modulo $p$ and $p \equiv 3$ modulo 4 , this equation has no non-trivial solutions; hence $\bar{\lambda} \equiv \delta_{0} \equiv 0$ modulo $p$, which is a contradiction.

Acknowledgements I am most grateful to Ben Moonen and Arne Smeets for their help and encouragement. I wish to thank Lie Fu for many useful discussions around the topics of this paper. I also thank the referee for his/her careful review.

Funding Open Access funding enabled and organized by Projekt DEAL.

Open Access This article is licensed under a Creative Commons Attribution 4.0 International License, which permits use, sharing, adaptation, distribution and reproduction in any medium or format, as long as you give appropriate credit to the original author(s) and the source, provide a link to the Creative Commons licence, and indicate if changes were made. The images or other third party material in this article are included in the article's Creative Commons licence, unless indicated otherwise in a credit line to the material. If material is not included in the article's Creative Commons licence and your intended use is not permitted by statutory regulation or exceeds the permitted use, you will need to obtain permission directly from the copyright holder. To view a copy of this licence, visit http://creativecommons.org/licenses/by/4.0/.

\section{References}

1. André, Y.: On the Shafarevich and Tate conjectures for hyper-kähler varieties. Math. Ann. 305(1), 205-248 (1996). https://doi.org/10.1007/BF01444219

2. André , Y.: Pour une théorie inconditionnelle des motifs. Publ. Math. l'IHÉS 83(1), 5-49 (1996). http:// www.numdam.org/item?id=PMIHES_1996_83_5_0

3. Amerik, E., Verbitsky, M.: Rational curves on hyperkähler manifolds. Int. Math. Res. Not. 2015(23), 13009-13045 (2015). https://doi.org/10.1093/imrn/rnv133 
4. Amerik, E., Verbitsky, M.: Collections of orbits of hyperplane type in homogeneous spaces, homogeneous dynamics, and hyper-kähler geometry. Int. Math. Res. Not. 2020(1), 25-38 (2020). https://doi.org/10. 1093/imrn/rnx319

5. Beauville, A.: Variétés Kählériennes dont la première classe de Chern est nulle. J. Differ. Geom. 18(4), 755-782 (1983). http://projecteuclid.org/euclid.jdg/1214438181

6. Borel, A.: Some metric properties of arithmetic quotients of symmetric spaces and an extension theorem. J. Differ. Geometry 6(4), 543-560 (1972). https://doi.org/10.4310/jdg/1214430642

7. Charles, F.: Birational boundedness for holomorphic symplectic varieties, Zarhin's trick for K3 surfaces, and the Tate conjecture. Ann. Math. 184(2), 487-526 (2016). https://doi.org/10.4007/annals.2016.184.2. 4

8. Deligne, P.: La conjecture de Weil pour les surfaces K3. Invent. Math. 15(3), 206-226 (1971). https://doi. org/10.1007/BF01404126

9. Floccari, S., Fu, L., Zhang, Z.: Erratum for “On the motive of O'Grady's ten-dimensional hyper-Kähler varieties" (2021). https://www.math.ru.nl/ liefu/articles/OG10-Erratum.pdf

10. Floccari, S., Fu, L., Zhang, Z.: On the motive of O’Grady's ten-dimensional hyper-Kähler varieties. Commun. Contemp. Math. 23, 04 (2021). https://doi.org/10.1142/S0219199720500340

11. Fu, L., Li, Z.: Supersingular irreducible symplectic varieties. In: Rationality of Algebraic Varieties, Schiermoonnikoog, 2019 (2021). https://arxiv.org/abs/1808.05851

12. Floccari, S.: On the Mumford-Tate conjecture for hyper-Kähler varieties. Manuscr. Math. (2021). https:// doi.org/10.1007/s00229-021-01316-4

13. Frei, S.: Moduli spaces of sheaves on K3 surfaces and Galois representations. Sel. Math. New Ser. 26(6), 1-16 (2020). https://doi.org/10.1007/s00029-019-0530-7

14. Gritsenko, V., Hulek, K., Sankaran, G.K.: Moduli spaces of irreducible symplectic manifolds. Compos. Math. 146(2), 404-434 (2010). https://doi.org/10.1112/S0010437X0900445X

15. Huybrechts, D.: Compact hyper-Kähler manifolds: basic results. Invent. Math. 135(1), 63-113 (1999). https://doi.org/10.1007/s002220050280

16. Huybrechts, D.: Finiteness results for compact hyperkähler manifolds. J. Reine Angew. Math. (Crelles J.) 2003(558), 15-22 (2006). https://doi.org/10.1515/crll.2003.038

17. Huybrechts, D.: A global Torelli theorem for hyper-Kähler manifolds [after M. Verbitsky]. Sémin. Bourbaki Juin 2011, Astérisque, 1040, 375-403 (2012). http://www.bourbaki.ens.fr/TEXTES/1040.pdf

18. Looijenga, E., Lunts, V.A.: A Lie algebra attached to a projective variety. Invent. Math. 129(2), 361-412 (1997). https://doi.org/10.1007/s002220050166

19. Markman, E.: On the monodromy of moduli spaces of sheaves on K3 surfaces. J. Algebr. Geom. 17(1), 29-99 (2008). https://doi.org/10.1090/S1056-3911-07-00457-2

20. Markman, E.: A survey of Torelli and monodromy results for holomorphic-symplectic varieties. In: Complex and Differential Geometry. Springer Proceedings of Mathematics, vol. 8, pp. 257-322. Springer, Berlin (2011). https://doi.org/10.1007/978-3-642-20300-8_15

21. Mongardi, G.: A note on the Kähler and Mori cones of hyper-Kähler manifolds. Asian J. Math. 19(4), 583-592 (2015). https://doi.org/10.4310/AJM.2015.v19.n4.a1

22. Moonen, B.: Families of motives and the Mumford-Tate conjecture. Milan J. Math. 85(2), 257-307 (2017). https://doi.org/10.1007/s00032-017-0273-x

23. Moonen, B.: On the Tate and Mumford-Tate conjectures in codimension 1 for varieties with $h^{2,0}=1$. Duke Math. J. 166(4), 739-799 (2017). https://doi.org/10.1215/00127094-3774386

24. O'Grady, K.: Desingularized moduli spaces of sheaves on a K3. J. Reine Angew. Math. 512, 49-117 (1999). https://doi.org/10.1515/crll.1999.056

25. O’Grady, K.: A new six-dimensional irreducible symplectic variety. J. Algebr. Geom. 12(3), 435-505 (2003). https://doi.org/10.1090/S1056-3911-03-00323-0

26. Serre, J.-P.: Local Fields, Graduate Texts in Mathematics, vol. 67. Springer, Berlin (1979). https://doi. org/10.1007/978-1-4757-5673-9

27. Soldatenkov, A.: On the Hodge structure of compact hyper-Kähler manifolds (2019). https://arxiv.org/ abs/1905.07793

28. Soldatenkov, A.: Deformation principle and André motives of projective hyper-Kähler manifolds. Int. Math. Res. Not. (2021). https://doi.org/10.1093/imrn/rnab108

29. Verbitsky, M.: Mirror symmetry for hyper-Kähler manifolds. AMS/IP Stud. Adv. Math. 10, 115-156 (1995)

30. Verbitsky, M.: Cohomology of compact hyper-Kähler manifolds and its applications. Geom. Funct. Anal. GAFA 6(4), 601-611 (1996). https://doi.org/10.1007/BF02247112

31. Verbitsky, M.: Mapping class group and a global Torelli theorem for hyper-Kähler manifolds. Duke Math. J. 162(15), 2929-2986 (2013). https://doi.org/10.1215/00127094-2382680 
32. Yang, Z.: On irreducible symplectic varieties of $\mathrm{K}^{[n]}$-type in positive characteristic (2019). https://arxiv. org/abs/1911.05653

Publisher's Note Springer Nature remains neutral with regard to jurisdictional claims in published maps and institutional affiliations. 